\title{
ELEMENTS GENERATING A PROPER NORMAL SUBGROUP OF THE CREMONA GROUP
}

\author{
SERGE CANTAT, VINCENT GUIRARDEL, AND ANNE LONJOU
}

\begin{abstract}
Consider an algebraically closed field $\mathbf{k}$, and let $\mathrm{Cr}_{2}(\mathbf{k})$ be the Cremona group of all birational transformations of the projective plane over $\mathbf{k}$. We characterize infinite order elements $g \in \mathrm{Cr}_{2}(\mathbf{k})$ having a power $g^{n}, n \neq 0$, generating a proper normal subgroup of $\mathrm{Cr}_{2}(\mathbf{k})$.
\end{abstract}

\section{INTRODUCTION}

Let $\mathbf{k}$ be a field. Let $\mathrm{Cr}_{2}(\mathbf{k})$ be the group of birational transformations of the projective plane $\mathbb{P}_{\mathbf{k}}^{2}$; we shall call it the Cremona group. If $f$ is an element of $\mathrm{Cr}_{2}(\mathbf{k})$, one can write $f$ in homogeneous coordinates $[x: y: z]$ as

$$
f[x: y: z]=[P: Q: R]
$$

where $P, Q$, and $R$ are three homogeneous polynomials of the same degree $d$ in $(x, y, z)$ with no common factor of positive degree: the integer $d$ is called the degree of $f$, and is denoted $\operatorname{deg}(f)$.

The Cremona group acts by isometries on a hyperbolic space of infinite dimension $\mathbb{H}_{\infty}$ (see Subsection 2.3). There are three types of isometries of such a space, and therefore also three types of elements $f \in \mathrm{Cr}_{2}(\mathbf{k})$ (see Section (3):

(1) elliptic isometries correspond to birational transformations for which the sequence $\left(\operatorname{deg}\left(f^{n}\right)\right)$ is bounded;

(2) parabolic isometries correspond to a polynomial growth of $\left(\operatorname{deg}\left(f^{n}\right)\right)$ : either $\operatorname{deg}\left(f^{n}\right)$ grows linearly and $f$ is called a Jonquières twist, or $\operatorname{deg}\left(f^{n}\right)$ grows quadratically and $f$ is called a Halphen twist;

(3) loxodromic isometries correspond to birational transformations for which $\operatorname{deg}\left(f^{n}\right)$ grows exponentially fast as $n$ goes to $+\infty$.

The name for Jonquières and Halphen twists come from the following properties. If $\mathbf{k}$ is algebraically closed, such a twist $f: \mathbb{P}_{\mathbf{k}}^{2} \rightarrow \mathbb{P}_{\mathbf{k}}^{2}$ preserves a unique

Date: January 6, 2020.

Key words and phrases. Cremona group; normal subgroups; Halphen twists; small cancellation.

The third author acknowledges support from the french Academy of Sciences (Fondation del Duca), and the third author from the Swiss National Science Foundation Grant "Birational transformations of threefolds" 200020_178807. 
pencil of curves, of genus 0 or 1 respectively. Moreover, every pencil of rational curves is equivalent, via a birational change of coordinates, to the pencil of lines through the point $[0: 0: 1]$, and the group of birational transformations preserving this pencil is known as the Jonquières group. And every pencil of curves of genus 1 is equivalent to a Halphen pencil (see Section 4).

A monomial transformation is a birational transformation that, in affine coordinates $(x, y)=[x: y: 1]$, can be written $f(x, y)=\left(x^{a} y^{b}, x^{c} y^{d}\right)$ for some integers $a, b, c$, and $d$. The group of all monomial transformations is isomorphic to $\mathrm{GL}_{2}(\mathbf{Z})$.

In a group $G$, we say that an element $h$ generates a proper normal subgroup if the smallest normal subgroup containing $h$ is not equal to $G$.

Theorem A. Let $\mathbf{k}$ be an algebraically closed field of characteristic 0 . Let $f$ be an element of $\mathrm{Cr}_{2}(\mathbf{k})$ of infinite order. The following properties are equivalent.

(a) For some $n \neq 0, f^{n}$ generates a proper normal subgroup of $\mathrm{Cr}_{2}(\mathbf{k})$.

(b) The birational transformation $f$ is a Halphen twist or a loxodromic element that is not conjugate to a monomial transformation.

A version of this theorem in positive characteristic is given in Section 3.4, Theorem A'. The proof of this theorem is a combination of several recent results together with one new input. Indeed, the heart of our article is Theorem B:

Theorem B. There exists a positive integer $n_{0}$ with the following property. Let $\mathbf{k}$ be a field. If $g \in \mathrm{Cr}_{2}(\mathbf{k})$ is a Halphen twist, then $g^{n_{0}}$ generates a proper normal subgroup of $\mathrm{Cr}_{2}(\mathbf{k})$. Moreover, this normal subgroup is a free product offree abelian groups of rank $\leq 8$.

The proof of Theorem B combines algebraic geometry with geometric group theory, as in [14] and [10]. In Theorem B, $n_{0}$ does not depend on the Halphen twist $g$, but in Theorem A, the integer $n$ must depend on $f$ : see Theorem 3.11. We refer to Theorem B', stated in Section 6.3, for a slightly stronger result.

Acknowledgement. Thanks to Julie Déserti for interesting discussions regarding Halphen pencils (parts of $\S 4$ are based on a joint work of the first author with Julie) and Stéphane Lamy for numerous discussions on normal subgroups of $\mathrm{Cr}_{2}(\mathbf{k})$. We are grateful to the referees for numerous interesting remarks.

\section{RATIONAL SURFACES AND HYPERBOLIC SPACES}

2.1. The lattice $\mathbf{Z}^{1, n}$. Let $n$ be a (maybe infinite) cardinal number; in what follows $n$ will be either a positive integer or the cardinality of the field $\mathbf{k}$.

Denote by $\mathbf{R}^{n}$ a real Hilbert space of dimension $n$, with a fixed orthonormal Hilbert basis $\left(\mathbf{e}_{j}\right)_{j \in J}$, where $J$ has cardinal $n$ (which may be uncountable). 
Then, consider a one dimensional space $\mathbf{R e}_{0}$, and define $\mathbf{R}^{1, n}$ to be the direct sum $\mathbf{R e}_{0} \oplus \mathbf{R}^{n}$, together with the (indefinite) scalar product defined by

$$
u \cdot v=a_{0} b_{0}-\sum_{j \in J} a_{j} b_{j}
$$

for every pair of vectors $u=a_{0} \mathbf{e}_{0}+\sum_{j} a_{j} \mathbf{e}_{j}$ and $v=b_{0} \mathbf{e}_{0}+\sum_{j} b_{j} \mathbf{e}_{j}$ with coefficients satisfying $\sum_{j} a_{j}^{2}<+\infty$ and $\sum_{j} b_{j}^{2}<+\infty$. We shall also set $u^{2}=u \cdot u$.

The subset $\mathbb{H}_{n} \subset \mathbf{R}^{1, n}$ is defined as the set of vectors $u$ in $\mathbf{R}^{1, n}$ with $u^{2}=1$ and $u \cdot \mathbf{e}_{0}>0$. With the distance dist defined by the formula

$$
\cosh (\operatorname{dist}(u, v))=u \cdot v
$$

$\mathbb{H}_{n}$ is isometric to the classical hyperbolic space of dimension $n$. Its boundary $\partial \mathbb{H}_{n}$ can be identified with the isotropic rays $\mathbf{R} \xi$ (where $\xi$ is any non-zero vector in $\mathbf{R}^{1, n}$ with $\xi^{2}=0$ ).

The lattice $\mathbf{Z}^{1, n}$ is, by definition, the subset of vectors $u=\sum_{i} a_{i} \mathbf{e}_{i} \in \mathbf{R}^{1, n}$ with integer coefficients $\left(a_{i}\right)$. The family $\left(\mathbf{e}_{i}\right)_{i \in I}$ is a basis of $\mathbf{Z}^{1, n}$ and the quadratic form associated to the scalar product satisfies

$$
\mathbf{e}_{0} \cdot \mathbf{e}_{0}=1, \mathbf{e}_{j} \cdot \mathbf{e}_{j}=-1 \text { for } 1 \leq j \leq n, \text { and } \mathbf{e}_{i} \cdot \mathbf{e}_{j}=0 \text { if } i \neq j .
$$

When $n$ is finite, this is the standard odd, unimodular quadratic form of signature $(1, n)$. To simplify the exposition, we shall write abusively $\mathbf{R}^{1, \infty}$ instead of $\mathbf{R}^{1, n}$ for any infinite $n$.

2.2. Blow-ups of the plane. Now, let $X$ be a rational surface that is obtained from $\mathbb{P}_{\mathbf{k}}^{2}$ by $n$ successive blow-ups; thus, $X$ comes with a birational morphism $\pi: X \rightarrow \mathbb{P}^{2}$. Denote by $\mathrm{NS}(X)$ the Néron-Severi group of $X$, by $\mathbf{e}_{0}$ the class of a line and by $\mathbf{e}_{i}, 1 \leq i \leq n$, the classes of the exceptional divisors (more precisely, $\mathbf{e}_{0}$ is the pull-back of the class of a line by $\pi$, and each $\mathbf{e}_{i}$ is the pull-back in $\mathrm{NS}(X)$ of the class of an exceptional divisor). The $\mathbf{e}_{i}$ form a basis of $\mathrm{NS}(X)$ and the intersection products between the $\mathbf{e}_{i}$ are exactly as in Equation (2.3). Thus, $\mathrm{NS}(X)$ is isometric to the lattice $\mathbf{Z}^{1, n}$, by a unique isometry identifying the two bases $\left(\mathbf{e}_{i}\right)$. Viewed in $\mathrm{NS}(X ; \mathbf{R})=\mathrm{NS}(X) \otimes \mathbf{R}$, the hyperbolic space $\mathbb{H}_{n}$ will be denoted by $\mathbb{H}_{X}$.

2.3. Infinitely many blow-ups. Blowing up all possible points of $\mathbb{P}_{\mathbf{k}}^{2}$, including infinitely near ones, one gets a nested family of rational surfaces $\pi: X \rightarrow$ $\mathbb{P}_{\mathbf{k}}^{2}$. The inductive limit of their Néron-Severi groups $\mathrm{NS}(X)$ is a well defined, infinite dimensional $\mathbf{Z}$-module $Z$; by definition, $Z$ is the Picard-Manin space. It comes with an intersection form and a natural basis $\left(\mathbf{e}_{i}\right)$, where $\mathbf{e}_{0}$ is the class of a line in the plane, and each $\mathbf{e}_{j}, j \neq 0$, is the class of the exceptional divisor of a point (in some rational surface $X \rightarrow \mathbb{P}_{\mathbf{k}}^{2}$ ). Their relative intersections satisfy the Equation (2.3). We refer to [8, 17, 26] for a detailed account on 
this construction (and the definition of the bubble space indexing the elements $\mathbf{e}_{j}$ of that basis). With the notation of Section $2.1, Z$ is isometric to a lattice $\mathbf{Z}^{1, \infty} \subset \mathbf{R}^{1, \infty}$; here, $\mathbf{R}^{1, \infty}$ is the direct sum of $\mathbf{R e}_{0}$ and of the $\ell^{2}$-completion of $\oplus_{i \neq 0} \mathbf{R e}_{i}$.

Now assume $\mathbf{k}$ to be algebraically closed. Since all points have been blownup to construct $Z$, all indeterminacy points of birational transformations are resolved, and the Cremona group acts by isometries on $Z$ and on the hyperbolic space $\mathbb{H}_{\infty} \subset \mathbf{R}^{1, \infty}$ (see [8, 26]):

Theorem 2.1. Let $\mathbf{k}$ be an algebraically closed field. The group $\mathrm{Cr}_{2}(\mathbf{k})$ acts faithfully by linear isometries for the intersection form on $Z \simeq \mathbf{Z}^{1, \infty}$. In particular, it acts faithfully by isometries on the infinite dimensional hyperbolic space $\mathbb{H}_{\infty} \subset \mathbf{R}^{1, \infty}$.

Remark 2.2. If $\mathbf{k}$ is not algebraically closed, we can choose an algebraic closure $\overline{\mathbf{k}}$ of $\mathbf{k}$, and embed $\mathrm{Cr}_{2}(\mathbf{k})$ into $\mathrm{Cr}_{2}(\overline{\mathbf{k}})$ to get a faithful action on an infinite dimensional hyperbolic space.

\section{TYPES OF BIRATIONAL TRANSFORMATIONS}

From Section 2.3 we know that the Cremona group $\mathrm{Cr}_{2}(\mathbf{k})$ acts faithfully by isometries on $Z$ (with respect to the intersection product) and on $\mathbb{H}_{\infty}$ (with respect to dist). Since there are three types of isometries for such a space, we obtain three types of birational transformations of the plane: elliptic, parabolic, and loxodromic elements of $\mathrm{Cr}_{2}(\mathbf{k})$. We shall divide the proof of Theorem A (and Theorem A' below) in three cases, according to the type of $f \in \mathrm{Cr}_{2}(\mathbf{k})$.

3.1. Elliptic elements. A birational transformation $f \in \mathrm{Cr}_{2}(\mathbf{k})$ is elliptic if and only if its orbits on $\mathbb{H}_{\infty}$ are bounded, if and only if the sequence $\left(\operatorname{deg}\left(f^{n}\right)\right)$ is bounded. In [4], Jérémy Blanc and Julie Déserti prove that any elliptic element $f$ of infinite order is conjugate to an element $f^{\prime}$ of $\mathrm{PGL}_{3}(\mathbf{k})$ (their proof, written for $\mathbf{k}=\mathbf{C}$, works over any algebraically closed field). We can now follow an argument of Marat Gizatullin (see [20], Lemma 2) which is also given in the book [11] of Dominique Cerveau and Julie Déserti. The smallest normal subgroup containing $f$ contains also $f^{\prime}$, and because $\mathrm{PGL}_{3}(\mathbf{k})$ is simple, it contains $\mathrm{PGL}_{3}(\mathbf{k})$. In particular, it contains the involution $\eta([x: y: z])=[-x$ : $-y: z]$. But $\eta$ is conjugate to $\sigma([x: y: z])=[y z: z x: x y]$ in $\operatorname{Cr}_{2}(\mathbf{k})$ and by Noether-Castelnuovo theorem $\sigma$ and $\mathrm{PGL}_{3}(\mathbf{k})$ generate $\mathrm{Cr}_{2}(\mathbf{k})$; thus the normal subgroup generated by $f$ is equal to $\mathrm{Cr}_{2}(\mathbf{k})$. This proves the following lemma, and therefore also Theorem A over arbitrary algebraically closed field for elliptic elements. 
Lemma 3.1. Let $\mathbf{k}$ be an algebraically closed field. Let $f$ be a non-trivial element of $\mathrm{PGL}_{3}(\mathbf{k})$ or an elliptic element of $\mathrm{Cr}_{2}(\mathbf{k})$ of infinite order. The smallest normal subgroup of $\mathrm{Cr}_{2}(\mathbf{k})$ containing $f$ is equal to $\mathrm{Cr}_{2}(\mathbf{k})$.

Remark 3.2. Lemma 3.1 requires $\mathbf{k}$ to be algebraically closed. When $\mathbf{k}$ is the field $\mathbf{R}$ of real numbers, the smallest normal subgroup generated by any element of $\mathrm{Cr}_{2}(\mathbf{R})$ is a proper normal subgroup of $\mathrm{Cr}_{2}(\mathbf{R})$ (see [30, Corollary 1.4]). The same result holds for perfect fields with at least one Galois extension of degree 8, for instance $\mathbf{Q}$; this follows from [24, Theorem C(2)].

3.2. Parabolic elements and Jonquières twists. By definition, a parabolic element of $\mathrm{Cr}_{2}(\mathbf{k})$ acts on $\mathbb{H}_{\infty}$ without fixed point, but with a unique fixed point on the boundary $\partial \mathbb{H}_{\infty}$. According to [15, 19], there are in fact two types of parabolic elements:

(1) the sequence $\left(\operatorname{deg}\left(f^{n}\right)\right)$ grows linearly, and in that case, one says that $f$ is a Jonquières twist.

(2) the sequence $\left(\operatorname{deg}\left(f^{n}\right)\right)$ grows quadratically and in that case, $f$ is called a Halphen twist.

This result holds over any field.

Let us assume that $f$ is a Jonquières twist first (we shall study Halphen twists in Section 4), and that $\mathbf{k}$ is algebraically closed. Then, it is proved in [15] that $f$ preserves a unique pencil of rational curves in $\mathbb{P}_{\mathbf{k}}^{2}$ (this result of Jeffrey Diller and Charles Favre is stated for the field of complex numbers, but the proofs apply to any algebraically closed field). In particular, $f$ is a Jonquières transformation: it is a Cremona transformation that preserves a pencil of rational curves in $\mathbb{P}_{\mathbf{k}}^{2}$. Up to conjugacy by an element of $\mathrm{Cr}_{2}(\mathbf{k})$, there is a unique pencil of rational curves in the plane, namely the pencil of lines through a point. Thus, $f$, as well as any Jonquières transformation, is conjugate to a birational transformation of the affine plane $\mathbb{A}_{\mathbf{k}}^{2}$ that permutes the vertical lines;

$$
f(x, y)=\left(A(x), B_{x}(y)\right)
$$

where $A$ is in $\mathrm{PGL}_{2}(\mathbf{k})$ and $B_{x}$ is in $\mathrm{PGL}_{2}(\mathbf{k}(x))$. Marat Gizatullin proved in [20, Lemma 2] that the smallest normal subgroup of $\mathrm{Cr}_{2}(\mathbf{k})$ containing such an element $f \neq$ Id coincides always with $\mathrm{Cr}_{2}$ (k) (see also [11, Prop. 5.21]). (1) Thus, we obtain the following lemma.

\footnotetext{
${ }^{1}$ The proof works as follows. Write $f$ as in Equation (3.1), and choose $C_{x} \in \mathrm{PGL}_{2}(\mathbf{k}(x))$ such that the transformation $h(x, y)=\left(x, C_{x}(y)\right)$ does not commute to $f$. Then, the commutator $[f, h]$ is an element of the simple group $\mathrm{PGL}_{2}(\mathbf{k}(x))$. This proves that the smallest normal subgroup containing $f$ contains $\mathrm{PGL}_{2}(\mathbf{k})$, and then one can apply Lemma 3.1 .
} 
Lemma 3.3. Let $\mathbf{k}$ be an algebraically closed field. If $f \in \mathrm{Cr}_{2}(\mathbf{k})$ is a Jonquières transformation (for instance a Jonquières twist), the smallest normal subgroup of $\mathrm{Cr}_{2}(\mathbf{k})$ containing $f$ coincides with $\mathrm{Cr}_{2}(\mathbf{k})$.

This proves Theorem A over any algebraically closed field when $f$ is a Jonquières twist, because all iterates $f^{n}, n \neq 0$, are again Jonquières twists.

Remark 3.4. Lemma 3.3 requires $\mathbf{k}$ be algebraically closed (see Remark 3.2).

Remark 3.5. Most elements of finite order in $\mathrm{Cr}_{2}(\mathbf{k})$ are conjugate to Jonquières transformations; as such, they do not generate a proper normal subgroup. When $\mathbf{k}=\mathbf{C}$, we know that there are 29 families of finite order elements which are not conjugate to Jonquières transformations, and each of them is conjugate to an automorphism of a del Pezzo surface of degree 1, 2 or 3 (see [3, Theorem 3]). It would be interesting to decide whether some of them may generate a proper normal subgroup of $\mathrm{Cr}_{2}(\mathbf{k})$.

3.3. Loxodromic transformations. An element $f$ of $\mathrm{Cr}_{2}(\mathbf{k})$ is loxodromic if it fixes two boundary points of $\mathbb{H}_{\infty}$ and acts as a non-trivial translation on the geodesic joining these two points. If $L(f)$ is the hyperbolic length of this translation, then the sequence $\left(\operatorname{deg}\left(f^{n}\right)\right)$ grows like $\exp (|n| L(f))$ as $n$ goes to $\pm \infty$. By definition, the number

$$
\lambda(f)=\exp (L(f))=\lim _{n \rightarrow+\infty} \operatorname{deg}\left(f^{n}\right)^{1 / n}
$$

is the dynamical degree of $f$ (see [7]).

3.3.1. p-Automorphisms (see [8, 28]). Before studying all loxodromic elements, we focus on a class of birational transformations that is defined only when the characteristic of $\mathbf{k}$ is positive. Note that Theorem 3.8 below has been obtained under slightly more restrictive hypotheses by Nicholas ShepherdBarron in a recent version of [28].

Assume that $\mathbf{k}$ is a field of characteristic $\operatorname{char}(\mathbf{k})=p>0$. Let $\operatorname{Fr}(t)=t^{p}$ be the Frobenius endomorphism of $\mathbf{k}$. An element $\varphi(t)$ of $\mathbf{k}[t]$ is a linearized polynomial or is a $p$-polynomial if all its monomials have degree $p^{j}$ for some $j \geq 0$; in other words, one can write

$$
\varphi(t)=\sum_{j} a_{j} t^{p^{j}}=\sum_{j} a_{j} \mathrm{Fr}^{j}(t)
$$

where $\operatorname{Fr}^{j}$ is the $j$-th iterate of the Frobenius endomorphism, i.e. $t^{p^{j}}=\operatorname{Fr}^{j}(t)=$ $\operatorname{Fr} \circ \operatorname{Fr} \circ \cdots \circ \operatorname{Fr}(t)$ ( $j$ compositions). These $p$-polynomials are exactly the polynomial transformations of $\mathbf{k}$ which are additive $\varphi(x+y)=\varphi(x)+\varphi(y)$. The composition of two $p$-polynomials $\varphi$ and $\psi: \mathbf{k} \rightarrow \mathbf{k}$ is another $p$-polynomial; 
with the laws given by addition and composition, the set of linearized polynomials $\mathbf{k}[\mathrm{Fr}]$ is, naturally, a non-commutative $\mathbf{k}$-algebra.

Let $\mathbb{G}_{a}$ denote the additive group of dimension 1 . Every $2 \times 2$ matrix with coefficients in $\mathbf{k}[\mathrm{Fr}]$ determines an algebraic endomorphism of the algebraic group $\mathbb{G}_{a}(\mathbf{k}) \times \mathbb{G}_{a}(\mathbf{k})$ : if $a, b, c$ and $d$ are the coefficients of the matrix, the endomorphism is given by

$$
f(x, y)=(a(x)+b(y), c(x)+d(y)) .
$$

It is invertible if and only if the matrix is invertible over the non-commutative $\operatorname{ring} \mathbf{k}[\mathrm{Fr}]$.

Lemma 3.6. Let $\mathbf{k}$ be a field of characteristic $p>0$. The group of algebraic automorphisms of the algebraic group $\mathbb{G}_{a}(\mathbf{k}) \times \mathbb{G}_{a}(\mathbf{k})$ coincides with the group $\mathrm{GL}_{2}(\mathbf{k}[\mathrm{Fr}])$.

Proof. Consider an algebraic automorphism $F$ of the algebraic group $\mathbb{G}_{a}(\mathbf{k}) \times$ $\mathbb{G}_{a}(\mathbf{k})$. Writing $F(x, y)=\left(U_{1}(x, y), U_{2}(x, y)\right)$, we see that $U_{i}$ satisfies the relation $U_{i}\left(x_{1}+x_{2}, y_{1}+y_{2}\right)=U_{i}\left(x_{1}, y_{1}\right)+U_{i}\left(x_{2}, y_{2}\right)$. Looking at the highest degree term $x^{k} y^{\ell}$ of $U_{i}$ (in the lexicographic order), one sees that it must be a power of $x$ or $y$, of degree $p^{m}$ for some $m$. Then, by induction on the degree, $U_{1}(x, y)=a(x)+b(y)$ and $U_{2}(x, y)=c(x)+d(y)$ for some pairs of linearized polynomials. Since $F$ is invertible, one concludes that $F$ is an element of $\mathrm{GL}_{2}(\mathbf{k}[\mathrm{Fr}])$.

Consider the group $\mathrm{T} \subset \mathrm{Cr}_{2}(\mathbf{k})$ consisting of all translations

$$
t_{u, v}(x, y)=(x+u, y+v) ;
$$

this group is isomorphic to $\mathbb{G}_{a}(\mathbf{k}) \times \mathbb{G}_{a}(\mathbf{k})$. We define the group pAut $\left(\mathbb{A}_{\mathbf{k}}^{2}\right)$ of $p$-automorphisms of the affine plane as the normalizer of $\mathrm{T}_{\text {in }} \mathrm{Cr}_{2}(\mathbf{k})$.

Lemma 3.7. The group $\mathrm{pAut}\left(\mathbb{A}_{\mathbf{k}}^{2}\right)$ is a subgroup of $\operatorname{Aut}\left(\mathbb{A}_{\mathbf{k}}^{2}\right)$, and

$$
\operatorname{pAut}\left(\mathbb{A}_{\mathbf{k}}^{2}\right)=\left(\mathbb{G}_{a}(\mathbf{k}) \times \mathbb{G}_{a}(\mathbf{k})\right) \rtimes \mathrm{GL}_{2}(\mathbf{k}[\mathrm{Fr}]) .
$$

Proof. Let $f \in \mathrm{Cr}_{2}(\mathbf{k})$ be an element that normalizes T; here, $f$ is viewed as a birational transformation of $\mathbb{A}_{\mathbf{k}}^{2}$. For every $(a, b) \in \mathbb{G}_{a}(\mathbf{k}) \times \mathbb{G}_{a}(\mathbf{k})$, there exists a unique $(\tilde{a}, \tilde{b}) \in \mathbb{G}_{a}(\mathbf{k}) \times \mathbb{G}_{a}(\mathbf{k})$ such that

$$
f \circ t_{a, b} \circ f^{-1}=t_{\tilde{a}, \tilde{b}} \text {. }
$$

The map $(a, b) \mapsto(\tilde{a}, \tilde{b})$ is the group-automorphism of $\mathbb{G}_{a}(\mathbf{k}) \times \mathbb{G}_{a}(\mathbf{k})$ determined by conjugacy by $f$. Equivalently, $f \circ t_{a, b}=t_{\tilde{a}, \tilde{b}} \circ f$. This equation shows that the set $\operatorname{Ind}(f) \subset \mathbb{A}_{\mathbf{k}}^{2}$ of indeterminacies of $f$ is invariant under translations, thus $\operatorname{Ind}(f)$ is empty, and $\operatorname{Ind}\left(f^{-1}\right)$ too by the same reasoning. So, $f$ is a regular automorphism of $\mathbb{G}_{a}(\mathbf{k}) \times \mathbb{G}_{a}(\mathbf{k})$. Changing $f$ into $f \circ t_{u, v}$ with 
$(u, v)=f^{-1}(0,0)$, we assume that $f$ fixes the origin. Evaluating Equation (3.6) at the origin, we get $(\tilde{a}, \tilde{b})=f(a, b)$. Thus, $f$ is now an algebraic automorphism of the group $\mathbb{G}_{a}(\mathbf{k}) \times \mathbb{G}_{a}(\mathbf{k})$, and the conclusion follows from Lemma 3.6 .

When we say that an element $q$ of $\mathbf{k}[\mathrm{Fr}]$ has degree $d$ (with respect to $\mathrm{Fr}$ ), we mean that $q=\sum_{j=0}^{d} q_{j} \operatorname{Fr}^{j}$ with $q_{d} \neq 0$; writing $\operatorname{Fr}^{j}(t)=t^{p^{j}}, q$ becomes an element of $\mathbf{k}[t]$ of degree $p^{d}$.

Let $\operatorname{Aff}\left(\mathbb{A}_{\mathbf{k}}^{2}\right)=\left(\mathbb{G}_{a}(\mathbf{k}) \times \mathbb{G}_{a}(\mathbf{k})\right) \rtimes \mathrm{GL}_{2}(\mathbf{k}[\mathrm{Fr}])$ be the group of affine transformations of the affine plane $\mathbb{A}_{\mathbf{k}}^{2}$, a subgroup of $\operatorname{pAut}\left(\mathbb{A}_{\mathbf{k}}^{2}\right)$. Let $\operatorname{El}\left(\mathbb{A}_{\mathbf{k}}^{2}\right)$ be the group of all elementary automorphisms, i.e. automorphisms

$$
h(x, y)=(u x+q(y), v y+w)
$$

with $q \in \mathbf{k}[y], u, v$, and $w$ in $\mathbf{k}$, and $u v \neq 0$. The subgroup $\operatorname{pEl}\left(\mathbb{A}_{\mathbf{k}}^{2}\right)=\operatorname{El}\left(\mathbb{A}_{\mathbf{k}}^{2}\right) \cap$ $\operatorname{pAut}\left(\mathbb{A}_{\mathbf{k}}^{2}\right)$ of elementary $p$-automorphisms consists of automorphisms $h$ as in Equation (3.7) with $q \in \mathbf{k}[\mathrm{Fr}]$.

Theorem 3.8. Let $\mathbf{k}$ be a field of characteristic $p>0$.

(1) The group $\operatorname{pAut}\left(\mathbb{A}_{\mathbf{k}}^{2}\right)$ is generated by $\operatorname{Aff}\left(\mathbb{A}_{\mathbf{k}}^{2}\right)$ and $\operatorname{pEl}\left(\mathbb{A}_{\mathbf{k}}^{2}\right)$. It is the amalgamated product of these two groups along their intersection.

(2) The dynamical degree of any p-automorphism is a non-negative power of $p$.

Proof. To ligthen notations, we set $A=\operatorname{Aff}\left(\mathbb{A}_{\mathbf{k}}^{2}\right), E=E I\left(\mathbb{A}_{\mathbf{k}}^{2}\right)$ and $p E=p E I\left(\mathbb{A}_{\mathbf{k}}^{2}\right)$. We first prove that $A$ and $p E$ generate $p A u t\left(\mathbb{A}_{\mathbf{k}}^{2}\right)$. By Lemma 3.7, it suffices to prove that $\mathrm{GL}_{2}(\mathbf{k}[\mathrm{Fr}]) \subset\langle\mathrm{A}, \mathrm{pE}\rangle$.

Consider $f \in \mathrm{GL}_{2}(\mathbf{k}[\mathrm{Fr}])$ with coefficients $a, b, c$, and $d$ in $\mathbf{k}[\mathrm{Fr}]$, as in Equation (3.4). If $c=0$, then $f(x, y)=(a(x)+b(y), d(y))$, and since $f$ is an automorphism of $\mathbb{A}_{\mathbf{k}}^{2}$ the degree of $a(t)$ and $d(t)$ with respect to $t$ must be equal to 1 . Thus, $f$ is an elementary $p$-automorphism, i.e. $f \in \mathrm{pE}$. If $a=0$, consider the linear automorphism $J \in A$ defined by $J(x, y)=(y, x)$. Then as above, $J \circ f \in \mathrm{pE}$ and we are done.

Assume now that $a c \neq 0$. Write $a=\sum_{i=0}^{m} a_{i} \mathrm{Fr}^{j}$ and $c=\sum_{j=0}^{n} c_{j} \mathrm{Fr}^{j}$ with $m=\operatorname{deg}(a)$ and $n=\operatorname{deg}(c)$ (the degrees are with respect to Fr). We argue by induction on the complexity $\operatorname{deg}(a)+\operatorname{deg}(c)$. Up to changing $f$ into $J \circ f$, we may assume that $m \leq n$. Set $u=c_{n}\left(\operatorname{Fr}^{n-m}\left(a_{m}\right)\right)^{-1}$, and compose $f$ with the p-automorphism

$$
g(x, y)=\left(x, y-u \mathrm{Fr}^{n-m}(x)\right) \in J \circ \mathrm{pE} \circ J^{-1}
$$

to get a new element $g \circ f$ of $\mathrm{GL}_{2}(\mathbf{k}[\mathrm{Fr}])$ with lower complexity. We conclude by induction that $g \circ f$ and therefore $f$ lie in $\langle\mathrm{A}, \mathrm{pE}\rangle$.

To conclude the proof of the first assertion, set $S=A \cap p E=A \cap E$; this is the group of affine automorphisms of $\mathbb{A}_{\mathbf{k}}^{2}$ whose linear part is upper triangular. By 
the theorem of Jung and van der Kulk, one has a decomposition into an amalgamated product $\operatorname{Aut}\left(\mathbb{A}_{\mathbf{k}}^{2}\right)=A * s E$. One can then conclude the proof of the first assertion using a reduced form argument in the amalgamated product, or arguing geometrically as follows. From Theorem 7 of [27], chapter $4, \operatorname{Aut}\left(\mathbb{A}_{\mathbf{k}}^{2}\right)$ acts on a tree $\mathcal{T}$, with a fundamental domain given by an edge $e=v_{1} v_{2}$, where the stabilizer of $e, v_{1}$ and $v_{2}$ are respectively S, A and E. Restricting this action to the subgroup $\mathrm{pAut}\left(\mathbb{A}_{\mathbf{k}}^{2}\right)$, the stabilizers of $e, v_{1}$ and $v_{2}$ in $\operatorname{pAut}\left(\mathbb{A}_{\mathbf{k}}^{2}\right)$ are respectively $\mathrm{S}, \mathrm{A}$ and $\mathrm{pE}$. The orbit of $e$ under $\mathrm{pAut}\left(\mathbb{A}_{\mathbf{k}}^{2}\right)$ is a subtree of $\mathcal{T}$, to which we can apply Theorem 6 of [27], chapter 4: this gives the amalgamated product structure we were looking for.

For the second assertion, take a $p$-automorphism $f$, and write it as a composition

$$
f=g_{\ell} \circ g_{\ell-1} \circ \cdots \circ g_{1}
$$

where each factor $g_{i}$ is an element of A or $\mathrm{pE}$. We say that such a decomposition is reduced if none of the $g_{i}$ lies in $\mathrm{S}$, and no two consecutive $g_{i}$ lie in the same factor. Every $p$-automorphism has such a reduced decomposition, unless it is an element of S. We say that it is cyclically reduced if, moreover, $g_{n}$ and $g_{1}$ are not both in $\mathrm{A}$ or $\mathrm{pE}$.

If $f$ lies in $\mathrm{pE}$ or $\mathrm{A}$, then the degree of $f^{n}$ is bounded by $\operatorname{deg}(f)$, so the dynamical degree of $f$ is $\lambda(f)=1$. If $f$ is written as a reduced composition of length $\geq 2$, Theorem 2.1 in [18] asserts that $\operatorname{deg}(f)=\prod_{i} \operatorname{deg}\left(g_{i}\right)$ (2). Conjugating $f$ in $\operatorname{pAut}\left(\mathbb{A}_{\mathbf{k}}^{2}\right)$, we can assume that this decomposition is cyclically reduced so that the composition

$$
f^{n}=\left(g_{\ell} \circ g_{\ell-1} \circ \cdots \circ g_{1}\right) \circ \cdots \circ\left(g_{\ell} \circ g_{\ell-1} \circ \cdots \circ g_{1}\right)
$$

is also reduced for all $n \geq 1$, so $\operatorname{deg}\left(f^{n}\right)=\prod_{i} \operatorname{deg}\left(g_{i}\right)^{n}=\operatorname{deg}(f)^{n}$. So $\lambda(f)=$ $\operatorname{deg}(f)$, and this number is a power of $p$ because $f$ is a $p$-automorphism.

3.3.2. A result of Shepherd-Barron. In [10], a criterion is given to show that (a large iterate of) a given loxodromic element $f$ of the Cremona group $\mathrm{Cr}_{2}(\mathbf{k})$ generates a proper normal subgroup of $\mathrm{Cr}_{2}(\mathbf{k})$. In [28], Shepherd-Barron proves that this criterion is satisfied by every loxodromic element $f$, except in two cases: when $f$ is conjugate to a monomial transformation, or when $\operatorname{char}(\mathbf{k})=$

\footnotetext{
${ }^{2}$ The proof, written over $\mathbf{R}$ or $\mathbf{C}$ in [18], applies verbatim over any field. The main remark is the following: start with an automorphism $h_{0}=\left(h_{0}^{x}(x, y), h_{0}^{y}(x, y)\right)$ that satisfies $\operatorname{deg}\left(h_{0}^{x}\right) \leq$ $\operatorname{deg}\left(h_{0}^{y}\right)=d_{0}$; after composition with an element $h_{1}$ of $\mathrm{pE} \backslash \mathrm{A}$ of degree $d \geq 2$, we get an automorphism $h_{1} \circ h_{0}=\left(h^{\prime x}, h^{\prime y}\right)$ such that $\operatorname{deg}\left(h^{\prime x}\right)=d d_{0}>\operatorname{deg}\left(h^{\prime y}\right)$, and composing with an element $h_{2}$ of A $\backslash \mathrm{pE}$ we obtain an automorphism $h_{2} \circ h_{1} \circ h_{0}=\left(h^{\prime \prime x}, h^{\prime \prime y}\right)$ such that $\operatorname{deg}\left(h^{\prime \prime x}\right) \leq$ $\operatorname{deg}\left(h^{\prime \prime y}\right)=d d_{0}$. Then, to prove Theorem 2.1 of [18], do an induction on the length $\ell$ of $f$ in Equation (3.9), starting with $g_{1}$ if it is in A, or with $g_{0}=$ Id otherwise.
} 
$p>0$ and $f$ is conjugate to a $p$-automorphism of the plane. These results prove one direction of the following theorem.

Theorem $\mathbf{C}$. Let $\mathbf{k}$ be an algebraically closed field, and let $f$ be a loxodromic element of $\mathrm{Cr}_{2}(\mathbf{k})$. The following properties are equivalent:

(a) there is an integer $\ell \geq 1$ such that $f^{\ell}$ generates a proper normal subgroup of $\mathrm{Cr}_{2}(\mathbf{k})$;

(b) $f$ is not conjugate to a monomial transformation or, when $p:=\operatorname{char}(\mathbf{k})>$ 0 , to a p-automorphism of the plane.

To conclude the proof of this theorem, one needs to prove that monomial transformations and $p$-automorphisms do not generate proper normal subgroups. Let us do it when $f \neq \mathrm{Id}$ is a $p$-automorphism. Write $f(x, y)=F(x, y) \circ t_{a, b}$ where $t_{a, b}$ is a translation. Since $f$ is loxodromic, $F$ is not the identity. Then, for every translation $t_{u, v}$ (as in Equation 3.5), one gets

$$
f \circ t_{u, v} \circ f^{-1} \circ t_{u, v}^{-1}=t_{u^{\prime}, v^{\prime}}
$$

where $\left(u^{\prime}+u, v^{\prime}+v\right)=F(u, v)$. One can choose $t_{u, v}$ in such a way that $t_{u^{\prime}, v^{\prime}} \neq \mathrm{Id}$, because $F$ is not the identity. This implies that the smallest normal subgroup containing $f$ contains a non-trivial element $t_{u^{\prime}, v^{\prime}}$ of $\mathrm{PGL}_{3}(\mathbf{k})$, and the conclusion follows from Lemma 3.1. The proof is the same for monomial transformations, replacing the translations $t_{u, v}$ by the diagonal transformations $d_{u, v}:(x, y) \mapsto$ $(u x, v y)$ with $u v \neq 0$ (i.e. the additive group $\mathbb{G}_{a} \times \mathbb{G}_{a}$ by the multiplicative group $\left.\mathbb{G}_{m} \times \mathbb{G}_{m}\right)$.

Remark 3.9. When the field is not algebraically closed, the theorem of ShepherdBarron may be stated as follows: let $f$ be a loxodromic element of $\mathrm{Cr}_{2}(\mathbf{k})$; if the normal subgroup generated by $f^{n}$ coïncides with $\mathrm{Cr}_{2}(\mathbf{k})$ for all $n \neq 0$, then $f$ normalizes a commutative algebraic subgroup $G \subset \mathrm{Cr}_{2}(\mathbf{k})$ such that $G(\mathbf{k})$ is infinite and $G$ becomes isomorphic to $\mathbb{G}_{a}^{2}$ or $\mathbb{G}_{m}^{2}$ on any algebraic closure of $\mathbf{k}$. In $\mathrm{Cr}_{2}(\mathbf{R})$, the derived subgroup is a subgroup of infinite index that contains all monomial transformations (this follows from [30, Theorem 1.1] because the group of monomial transformations is generated by elements of $\operatorname{Bir}\left(\mathbb{P}_{\mathbf{k}}^{2}\right)$ of degree $\leq 2)$. If $\mathbf{k}$ is any perfect field with an algebraic Galois extension of degree 8 (for instance any number field), Stéphane Lamy and Susanna Zimmermann show that the smallest normal subgroup of $\mathrm{Cr}_{2}(\mathbf{k})$ containing any given monomial transformation is a proper subgroup of $\mathrm{Cr}_{2}(\mathbf{k})([24])$.

3.3.3. An example. Let $m$, and $q$ be positive integers. Set $n=m^{2}-1$. Consider the element $h$ of $\mathrm{Cr}_{2}(\mathbf{k})$ defined in affine coordinates by

$$
h(x, y)=\left(y, x+y^{e}\right)
$$


with $e=m$ modulo $n$. Let $\alpha$ be a root of unity such that $\alpha^{n}=1$ (i.e. $\alpha^{m^{2}}=\alpha$ ). Set $\beta=\alpha^{m}$ and consider the diagonal linear automorphism $g(x, y)=(\alpha x, \beta y)$. Then

$$
h \circ g \circ h^{-1}=g^{m} .
$$

Now, compose $h$ with a monomial transformation $f(x, y)=\left(x^{a} y^{b}, x^{c} y^{d}\right)$. Then, $(f \circ h) \circ g \circ(f \circ h)^{-1}=g^{q}$ if and only if the integers $a, b, c$, and $d$ satisfy

$$
a m+b=q \quad \text { and } \quad c m+d=q m \quad \bmod (n) .
$$

We can choose $c=q$ and $d=0$ modulo $n$, and then $b$ such that $b q= \pm 1$ modulo $n$ (this is possible if $q$ is invertible modulo $n$ ). Since $m$ is invertible modulo $n$, we then set $a=(q-b) / m$ modulo $n$. Thus, the system of congruences (3.14) has solutions for which $a d-b c= \pm 1$ modulo $n$; then, one can lift these solutions to elements of $\mathrm{GL}_{2}(\mathbf{Z})$. One gets more solutions by composition with elements in the kernel of the projection $\mathrm{GL}_{2}(\mathbf{Z}) \rightarrow \mathrm{GL}_{2}(\mathbf{Z} / n \mathbf{Z})$.

Proposition 3.10. Let $\mathbf{k}$ be a field. Let $m$ and $q$ be positive integers, set $n=$ $m^{2}-1$, and assume that $q$ is invertible modulo $n$. Then, there is a loxodromic element $s \in \mathrm{Cr}_{2}(\mathbf{k})$ and an elliptic element $g \in \mathrm{PGL}_{3}(\mathbf{k}) \subset \mathrm{Cr}_{2}(\mathbf{k})$ such that

(1) $g$ is of order $n$;

(2) $s \circ g \circ s^{-1}=g^{q}$;

(3) $s$ is not conjugate to a monomial map or a p-automorphism (if $\operatorname{char}(\mathbf{k})=$ $p>0)$.

Sketch of proof. Take $f, g$, and $h$ as above, and set $s=f \circ h$. All we need to show is that one can choose $f$ in such a way that $s$ is not conjugate to a monomial map or a $p$-automorphism. Composing $f$ with monomial maps given by matrices $M \in \mathrm{GL}_{2}(\mathbf{Z})$ which are equal to Id modulo $n$, and choosing $e$ large, we may assume that the entries of $f$ satisfy $e>c>d>a>b>1$. Then, a simple recursion shows that the degree of $(f \circ h)^{k}$ is equal to $(c+d e)^{k}$; in particular, the dynamical degree of $f \circ h$ is equal to the integer $c+d e$. Thus, $f \circ h$ is not conjugate to a monomial map because the dynamical degree of a loxodromic monomial map is not an integer: it is a quadratic unit. And, changing $e$ if necessary, one sees that $f \circ h$ is not conjugate to a $p$-automorphism because the dynamical degree of such an automorphism of the plane is a power of $p$ (see Theorem 3.8).

Pick such a pair of elements $(s, g)$ in the Cremona group. The smallest normal subgroup generated by $s^{k}$ contains

$$
\left[s^{k}, g\right]=s^{k} \circ g \circ s^{-k} \circ g^{-1}=g^{q^{k}-1} .
$$


Thus, if $q^{k} \neq 1$ modulo $n$ for all $1 \leq k \leq \ell$, the smallest normal subgroup generated by $s^{k}$, for any $k \leq \ell$, contains a non-trivial elliptic element and coïncides with $\mathrm{Cr}_{2}(\mathbf{k})$ if $\mathbf{k}$ is algebraically closed (Lemma 3.1). We obtain:

Theorem 3.11. Let $\mathbf{k}$ be an algebraically closed field. For every integer $\ell$, there is a loxodromic element $s$ in $\mathrm{Cr}_{2}(\mathbf{k})$ such that $s^{n}$ generates a proper normal subgroup of $\mathrm{Cr}_{2}(\mathbf{k})$ for some $n>\ell$, but not for $n \leq \ell$.

This result shows that, unlike in Theorem $\mathrm{B}$, one cannot take $n$ independent of $f$ in Theorem A and Theorem A' below.

3.4. Theorem A'. We can now state the extension of Theorem A' to algebraically closed fields of arbitrary characteristic.

Theorem A'. Let $\mathbf{k}$ be an algebraically closed field. Let $f$ be an element of $\mathrm{Cr}_{2}(\mathbf{k})$ of infinite order. The following properties are equivalent.

(a) There exists an integer $n>0$ such that $f^{n}$ generates a non-trivial proper normal subgroup of $\mathrm{Cr}_{2}(\mathbf{k})$.

(b) The birational transformation $f$ is a Halphen twist or it is a loxodromic element that is not conjugate to a monomial transformation or to a $p$ automorphism when $p:=\operatorname{char}(\mathbf{k})>0$.

We already proved this result for elements $f \in \mathrm{Cr}_{2}(\mathbf{k})$ which are not Halphen twists. The rest of this paper is devoted to this last case.

\section{HALPHEN TWISTS, HALPHEN PENCILS, AND TRANSLATION LENGTHS}

By definition, a Halphen twist $f$ in $\mathrm{Cr}_{2}(\mathbf{k})$ is a birational transformation $f: \mathbb{P}_{\mathbf{k}}^{2} \rightarrow \mathbb{P}_{\mathbf{k}}^{2}$ such that $\operatorname{deg}\left(f^{n}\right)$ grows quadratically. In this section, we describe a result of Marat Gizatullin showing that such a birational transformation preserves a unique pencil of curves of genus 1 . But before that, we start with a description of all such pencils and their geometry.

4.1. Horoballs. As in Section 2.1, consider the space $\mathbf{R}^{1, n}$, together with its lattice $\mathbf{Z}^{1, n}$ and hyperbolic subset $\mathbb{H}_{n}$. Assume that the vector $\xi=a_{0} \mathbf{e}_{0}-$ $\sum_{j} a_{j} \mathbf{e}_{j}$ of $\mathbf{R}^{1, n}$ satisfies $a_{0}>0$, and $\xi \cdot \xi=a_{0}^{2}-\sum_{j} a_{j}^{2}=0$. Then, $\xi$ determines a boundary point of the hyperbolic space $\mathbb{H}_{n}$. Let $\varepsilon$ be a positive real number. The horoball $H_{\xi}(\varepsilon)$ in $\mathbb{H}_{n}$ is the subset

$$
H_{\xi}(\varepsilon)=\left\{v \in \mathbb{H}_{n} ; 0<v \cdot \xi \leq \varepsilon\right\} .
$$

It is a limit of balls with centers converging to the boundary point $\xi$. The horosphere is the boundary

$$
\partial H_{\xi}(\varepsilon)=\left\{v \in \mathbb{H}_{n} ; v \cdot \xi=\varepsilon\right\} .
$$


Remark 4.1. If $h$ is any isometry of $\mathbb{H}_{n}$, then

$$
h\left(H_{\xi}(\varepsilon)\right)=H_{h(\xi)}(\varepsilon) .
$$

If $h$ fixes the boundary point $\mathbf{R} \xi \in \partial \mathbb{H}_{n}$ then $h$ maps $H_{\xi}(\varepsilon)$ to $H_{\xi}\left(e^{ \pm L(h)} \varepsilon\right)$, where $L(h)$ is the translation length of $h$ and the sign is positive (resp. negative) when $\xi$ is attracting (resp. repelling).

4.2. The lattice $\mathbf{Z}^{1,9}$ and the group $W_{9}$. Consider the lattice $\mathbf{Z}^{1, n}$ introduced in Subsection 2.1, but now for the specific dimension $n=9$. The anti-canonical vector $\xi_{9}=3 \mathbf{e}_{0}-\sum_{j=1}^{9} \mathbf{e}_{i}$ is isotropic, and the ray $\mathbf{R} \xi_{9}$ determines a boundary point of the hyperbolic space $\mathbb{H}_{9}$. The horoballs centered at this boundary point are the subsets $H_{\xi_{9}}(\varepsilon)=\left\{u \in \mathbb{H}_{9} ; 0<u \cdot \xi_{9} \leq \varepsilon\right\}$. The riemannian metric induced on $\partial H_{\xi_{9}}(\varepsilon)$ by the hyperbolic metric is euclidean, and we can identify

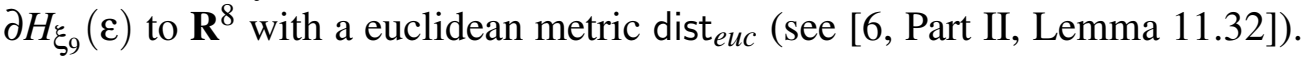
The euclidean and hyperbolic distances satisfy

$$
\frac{1}{2} \operatorname{dist}_{\text {euc }}(x, y)=\sinh \left(\frac{1}{2} \operatorname{dist}(x, y)\right)
$$

for all pairs of points $x$ and $y$ on $\partial H_{\xi_{9}}(\varepsilon)$ (see [29][§13] for instance).

Remark 4.2. The horospheres $\partial H_{\xi_{9}}(\varepsilon)$ foliate $\mathbb{H}_{9}$ and $\partial H_{\xi_{9}}(3)$ is the leaf containing $\mathbf{e}_{0}$. The geodesics of $\mathbb{H}_{9}$ with one end point equal to $\xi_{9}$ form a transverse foliation; if we follow this geodesic foliation, we get a family of (holonomy) maps $h_{\varepsilon}: \partial H_{\xi_{9}}(3) \rightarrow \partial H_{\xi_{9}}(\varepsilon)$. In the half-space model with $\xi_{9}$ at infinity, the horospheres are horizontal hyperplanes and the $h_{\varepsilon}$ are vertical translations. They are not isometric, but are similitudes with respect to the euclidean metrics on the horospheres: $h_{\varepsilon}$ multiplies euclidean distances by $\varepsilon / 3$.

In the orthogonal group $\mathrm{O}\left(\mathbf{Z}^{1,9}\right)$, there is an index 2 subgroup $\mathrm{O}\left(\mathbf{Z}^{1,9}\right)^{+}$preserving $\mathbb{H}_{9}$, and thus acting by isometries on $\mathbb{H}_{9}$. The subgroup $\mathrm{O}\left(\mathbf{Z}^{1,9} ; \xi_{9}\right)^{+}$ fixing the isotropic line $\mathbf{R} \xi_{9}$ fixes also the class $\xi_{9}$, because it is the unique primitive integral vector on this line with $a_{0}>0$; this group preserves every horosphere $\partial H_{\xi_{9}}(\varepsilon)$, acting on it as a group of affine isometries for the euclidean metric dist $_{e u c}$. The action of $\mathrm{O}\left(\mathbf{Z}^{1,9} ; \xi_{9}\right)^{+}$on $\partial H_{\xi_{9}}(3)$ is conjugate to the action on $\partial H_{\xi_{9}}(\varepsilon)$ by the similitude $h_{\varepsilon}$.

The group of affine isometries of the euclidean space $\partial H_{\xi_{9}}(\varepsilon)$ is an extension of an orthogonal group by its group of translations. Since the group $\mathrm{O}\left(\mathbf{Z}^{1,9} ; \xi_{9}\right)^{+}$is a discrete subgroup of this group of isometries, Bieberbach theorem shows that there is a finite index subgroup $P^{*}$ in $\mathrm{O}\left(\mathbf{Z}^{1,9} ; \xi_{9}\right)^{+}$acting by translations on the horosphere $\partial H_{\xi_{9}}(3)$. For $g$ in $P^{*}$, the translation length on $\partial H_{\xi_{9}}(3)$ with respect to dist euc $_{\text {is equal to dist }}$ euc $\left(\mathbf{e}_{0}, g\left(\mathbf{e}_{0}\right)\right)$. And if $g \in P^{*} \backslash\{\operatorname{Id}\},\left\langle\mathbf{e}_{0}, g\left(\mathbf{e}_{0}\right)\right\rangle$ is an integer $\geq 2$, so the hyperbolic distance satisfies 
$\operatorname{dist}\left(\mathbf{e}_{0}, g\left(\mathbf{e}_{0}\right)\right) \geq \operatorname{arcosh}(2)$; in view of Equation (4.3), the euclidean distance is bounded by

$$
\operatorname{dist}_{\text {euc }}\left(\mathbf{e}_{0}, g\left(\mathbf{e}_{0}\right)\right) \geq 2 \sinh \left(\frac{1}{2} \operatorname{arcosh}(2)\right)=\sqrt{2} .
$$

Since the conjugacy $h_{\varepsilon}$ is a similitude that multiplies distances by $\varepsilon / 3$, the group $P^{*}$ acts also by translations on each of the horospheres $\partial H_{\xi_{9}}(\varepsilon)$, with euclidean translation length bounded from below by

$$
\Delta_{\text {euc }}(\varepsilon):=\frac{\varepsilon \sqrt{2}}{3} .
$$

In terms of the hyperbolic metric, this says that for all $x \in \partial H_{\xi_{9}}(\varepsilon)$ and all $g \in P^{*} \backslash\{\mathrm{Id}\}$ one has

$$
\operatorname{dist}(x, g x) \geq \Delta(\varepsilon):=2 \operatorname{arcsinh}\left(\frac{\varepsilon}{3 \sqrt{2}}\right) .
$$

Denoting by $P_{n}^{*}$ the subgroup of $P^{*}$ consisting of all $n$-th powers of elements of $P^{*}$, one gets the following result.

Lemma 4.3. There is a finite index, normal subgroup $P^{*} \subset \mathrm{O}\left(\mathbf{Z}^{1,9} ; \xi_{9}\right)^{+}$with the following properties. For every $\varepsilon>0$, there is a positive real number $\Delta(\varepsilon)$, such that

(1) the group $P^{*}$ is isomorphic to the abelian group $\mathbf{Z}^{8}$;

(2) it acts by translations on the euclidean horosphere $\partial H_{\xi_{9}}(\varepsilon)$;

(3) the translation length of any element of $P_{n}^{*} \backslash\{\mathrm{Id}\}$ on $\partial H_{\xi_{9}}(\varepsilon)$ for the euclidean distance is at least $n \Delta_{\text {euc }}(\varepsilon)=n \varepsilon \sqrt{2} / 3$ and for all $x \in \partial H_{\xi_{9}}(\varepsilon)$, $\operatorname{dist}(x, g x) \geq \Delta(n \varepsilon)=2 \operatorname{arcsinh}\left(n \frac{\varepsilon}{3 \sqrt{2}}\right)$.

Remark 4.4 (See [9]). One can, in fact, be much more precise. The orthogonal complement of the vector $\xi_{9}$ is a sublattice $\mathbf{E}_{9} \subset \mathbf{Z}^{1,9}$. A basis of $\mathbf{E}_{9}$ is formed by the vectors $\alpha_{0}=\mathbf{e}_{0}-\mathbf{e}_{1}-\mathbf{e}_{2}-\mathbf{e}_{3}$ and $\alpha_{i}=\mathbf{e}_{i}-\mathbf{e}_{i+1}$, for $i=1, \ldots$, 8. The intersection matrix $\left(\alpha_{i} \cdot \alpha_{j}\right)$ is equal to $\Gamma_{9}-2 \mathrm{Id}_{9}$, where $\Gamma_{9}$ is the incidence matrix of the Dynkin diagram of type $E_{9}$. In particular, each class $\alpha_{i}$ has self- intersection -2 and determines an involutive isometry of $\mathbf{Z}^{1,9}$, namely $s_{i}: x \mapsto x+\left(x \cdot \alpha_{i}\right) \alpha_{i}$. By definition, these involutions generate the Weyl (or Coxeter) group $W_{9}$, and one shows that $W_{9}$ has finite index in $\mathrm{O}\left(\mathbf{Z}^{1,9} ; \xi_{9}\right)^{+}$. When restricted to $\mathbf{E}_{9}$, the intersection form is degenerate; its radical is generated by the vector $\xi_{9}$ and the lattice $\mathbf{E}_{8} \simeq \mathbf{E}_{9} / \mathbf{Z} \xi_{9}$ is isomorphic to the root lattice of finite type $E_{8}$. Then, $W_{9}$ is isomorphic to the affine Weyl group of type $E_{8}$, and fits in the extension $0 \rightarrow \mathbf{E}_{8} \rightarrow W_{9} \rightarrow W_{8} \rightarrow 1$ where the injection $\imath: \mathbf{E}_{8} \rightarrow W_{9}$ of the additive group $\mathbf{E}_{8} \simeq \mathbf{Z}^{8}$ into $W_{9}$ is defined by the following 
formula: for $w \in \mathbf{E}_{8}$,

$$
\mathfrak{l}(w): v \in \mathbf{Z}^{1,9} \mapsto v-\left(v \cdot \xi_{9}\right) w+\left((w \cdot v)-\frac{1}{2}\left(v \cdot \xi_{9}\right)(w \cdot w)\right) \xi_{9}
$$

The quotient $W_{8}$ is a finite reflection group. In Lemma 4.3, one can take $P^{*}=$ $\imath\left(\mathbf{E}_{8}\right)$.

\subsection{Halphen pencils and Halphen surfaces (see [16, 9]). A Halphen pencil} $\mathfrak{p}$ of index $m$ is an irreducible pencil of plane curves of degree $3 m$ with 9 basepoints of multiplicity $\geq m$; here, infinitely near points may be included in the list of base-points. In particular, a Halphen pencil of index 1 is made of cubic curves with no fixed component. A smooth rational surface $X$ is a Halphen surface if there exists an integer $m>0$ such that the linear system $\left|-m K_{X}\right|$ is of dimension 1, has no fixed component, and has no base-point (here, $K_{X}$ denotes the canonical divisor). The index of a Halphen surface is the smallest possible value for such a positive integer $m$.

As shown in [9], every Halphen surface $X$ is obtained by blowing-up the nine base-points of a Halphen pencil $\mathfrak{p}$. In particular, the Picard number of $X$ is equal to 10: there is a basis of the Néron-Severi group given by the class $\mathbf{e}_{0}$ of a line in $\mathbb{P}^{2}$, and the classes $\mathbf{e}_{j}$ of the 9 exceptional divisors (coming from the 9 base-points). The pencil $\mathfrak{p}$ determines a fibration $\pi: X \rightarrow \mathbb{P}^{1}$; this fibration is the same as the one given by the linear system $\left|-m K_{X}\right|$. Two cases may appear: if the general fiber of $\pi$ is a smooth curve of genus 1 we say that $\pi$ is elliptic; otherwise, the general fiber is a rational curve with a cusp and one says that $\pi$ is quasi-elliptic. Quasi-elliptic examples occur only in characteristic 2 and 3 (see the first sections of [5] or the last chapter of [12]); the cusps of the fibers form a smooth curve which will be denoted by $\Sigma_{X} \subset X$ (computing the intersection of $\Sigma_{X}$ with a fiber, one sees that $m$ divides $p$, so $m \leq 3$ ).

When $m>1$, the pencil contains a unique cubic curve with multiplicity $m$. The class of this curve in the Picard-Manin space is the class of $-K_{X}$ and it is equal to $\xi_{X}=3 \mathbf{e}_{0}-\mathbf{e}_{1}-\ldots-\mathbf{e}_{9}$; if we want to refer to the pencil, rather than to the surface, we shall denote this class by $\xi_{\mathfrak{p}}$ (it may be considered as a point of $\mathrm{NS}(X)$ or of $Z$ ). Thus, $\xi_{X}$ corresponds to the anti-canonical vector $\xi_{9}$ under the natural isometry $\mathbf{Z}^{1,9} \rightarrow \mathrm{NS}(X)$. The model $\mathbf{Z}^{1,9}$ does not depend on the pencil $\mathfrak{p}$ (or on the Halphen surface $X$ ).

The group of automorphisms $\operatorname{Aut}(X)$ acts on $\mathrm{NS}(X)$ and its image is a group of isometries fixing the anti-canonical class $\xi_{X}$. Since $\operatorname{Aut}(X)$ preserves the canonical bundle, it permutes the sections of $\left|-m K_{X}\right|$, and it preserves the fibration $\pi$ (permuting its fibers). 
Consider the group $\operatorname{Bir}\left(\mathbb{P}_{\mathbf{k}}^{2} ; \mathfrak{p}\right)$ of birational transformations of the plane preserving the pencil $\mathfrak{p}$. After conjugacy by the blow-up $X \rightarrow \mathbb{P}_{\mathbf{k}}^{2}, \operatorname{Bir}\left(\mathbb{P}_{\mathbf{k}}^{2} ; \mathfrak{p}\right)$ becomes a subgroup of $\operatorname{Bir}(X)$ that permutes the fibers of $\pi$. The fibration $\pi$ is relatively minimal, which amounts to say that there is no exceptional divisor of the first kind in the fibers of $\pi$ (see [21]); this implies that $\operatorname{Bir}\left(\mathbb{P}_{\mathbf{k}}^{2} ; \mathfrak{p}\right.$ ) is contained in $\operatorname{Aut}(X)$ (see [23]). Since Aut $(X)$ permutes the fibers of $\pi$, we conclude that $\operatorname{Bir}\left(\mathbb{P}_{\mathbf{k}}^{2} ; \mathfrak{p}\right)=\operatorname{Aut}(X)$ (after conjugacy by the blow-up $X \rightarrow \mathbb{P}^{2}$ ):

Lemma 4.5. Let $\mathfrak{p}$ be a Halphen pencil. Let $X$ be the Halphen surface and $\pi: X \rightarrow \mathbb{P}_{\mathbf{k}}^{1}$ be the fibration defined by $\mathfrak{p}$. Then Aut $(X)$ permutes the fibers of $\pi$, and is conjugate to $\operatorname{Bir}\left(\mathbb{P}_{\mathbf{k}}^{2} ; \mathfrak{p}\right)$ by the blow-up of the nine base-points of $\mathfrak{p}$.

Let $P_{0}(X) \subset \operatorname{Aut}(X)$ (resp. $P_{0}(\mathfrak{p}) \subset \operatorname{Bir}\left(\mathbb{P}_{\mathbf{k}}^{2}, \mathfrak{p}\right)$ ) be the subgroup of automorphisms $f$ such that the action $f^{*}: \mathrm{NS}(X) \rightarrow \mathrm{NS}(X)$ corresponds to an element of the abelian group $P^{*} \subset \mathrm{O}\left(\mathbf{Z}^{1,9}\right)^{+}$given by Lemma 4.3.

In the following lemma, $\xi_{X}$ is viewed as a boundary point of $\mathbb{H}_{\infty}$, via the natural embedding $\mathrm{NS}(X) \rightarrow Z$, the horosphere $\partial H_{\xi_{X}}(\varepsilon)$ is the horosphere in $\mathbb{H}_{\infty}$ centered at $\xi_{X}$, and the real number $\Delta(\varepsilon)$ is defined in Equation (4.6).

Lemma 4.6. The group $P_{0}(\mathfrak{p})$ is a normal subgroup of $\operatorname{Bir}\left(\mathbb{P}_{\mathbf{k}}^{2} ; \mathfrak{p}\right)$. For every $\varepsilon>0$, every non-trivial element $f$ of this group acts

- on $\partial H_{\xi_{X}}(\varepsilon) \cap \mathbb{H}_{X}$ as a euclidean translation, of length $\geq \Delta_{\text {euc }}(\varepsilon)$;

- on $\partial H_{\xi_{X}}(\varepsilon) \subset \mathbb{H}_{\infty}$ as a fixed point free isometry that satisfies

$$
\operatorname{dist}(u, f(u)) \geq \Delta(\varepsilon)
$$

for every $u \in \partial H_{\xi_{X}}(\varepsilon)$.

Moreover, for each $l>0$, there exists an integer $n \geq 1$ that does not depend on the pencil $\mathfrak{p}$, such that

$$
\operatorname{dist}_{\mathbb{H}_{\infty}}\left(x, g^{n}(x)\right) \geq l
$$

for each $g \in P_{0}(\mathfrak{p}) \backslash\{1\}$ and each point $x$ in the horosphere $\partial H_{\xi_{X}}(\varepsilon)$, .

Proof. The first property follows directly from the definition of $P_{0}(\mathfrak{p})$. For the second one, we use that $\partial H_{\xi_{X}}(\varepsilon) \cap \mathbb{H}_{X}$ is a $\operatorname{Bir}\left(\mathbb{P}_{\mathbf{k}}^{2} ; \mathfrak{p}\right)$-invariant euclidean subspace of the (infinite dimensional) euclidean space $\partial H_{\xi_{X}}(\varepsilon)$, so the euclidean orthogonal projection commutes with $\operatorname{Bir}\left(\mathbb{P}_{\mathbf{k}}^{2} ; \mathfrak{p}\right)$. Since it is 1-Lipschitz, it follows that $\operatorname{dist}_{\text {euc }}(u, f(u)) \geq \Delta_{\text {euc }}(\varepsilon)$ for all $u \in \partial H_{\xi_{X}}(\varepsilon)$. By Equation (4.3), the hyperbolic distance satisfies $\operatorname{dist}(u, f(u)) \geq \Delta(\varepsilon)$. The "moreover part" is now a consequence of Lemma 4.3 and $\Delta(n \varepsilon) \rightarrow \infty$ as $n \rightarrow \infty$.

4.4. Gizatullin's theorem. The following result encapsulates a theorem of Marat Gizatullin (see [19]) and results of Georges H. Halphen and Igor Dolgachev (see [16]). 
Theorem 4.7. There is an integer $B_{H} \leq 8$ ! with the following property. Let $\mathbf{k}$ be an algebraically closed field. Let $f \in \mathrm{Cr}_{2}(\mathbf{k})$ be a Halphen twist. Then, up to conjugacy, $f$ preserves a unique Halphen pencil $\mathfrak{p}$ (of some index $m \geq 1$ ), and:

- this Halphen pencil provides an elliptic fibration of the corresponding Halphen surface $X$,

- the kernel of the homomorphism $\operatorname{Aut}(X) \rightarrow \mathrm{GL}(\mathrm{NS}(X))$ is finite, of or$\operatorname{der} \leq B_{H}$.

In other words, one can conjugate $f$ by a birational transformation $g: \mathbb{P}_{\mathbf{k}}^{2}-\rightarrow$ $\mathbb{P}_{\mathbf{k}}^{2}$ and find a Halphen pencil $\mathfrak{p}$, of some index $m$, such that $f^{\prime}:=g \circ f \circ g^{-1}$ is contained in $\operatorname{Bir}\left(\mathbb{P}_{\mathbf{k}}^{2} ; \mathfrak{p}\right)$. Blowing-up the base-points of $\mathfrak{p}, f^{\prime}$ becomes an automorphism of a rational surface $X$, preserving a relatively minimal fibration $\pi: X \rightarrow \mathbb{P}_{\mathbf{k}}^{1}$ of genus 1 . The class of a general fiber is equal to $m \xi_{X}$, where $\xi_{X}=\xi_{\mathfrak{p}}=3 \mathbf{e}_{0}-\mathbf{e}_{1}-\ldots-\mathbf{e}_{9}$. Viewed in $\mathbb{H}_{\infty}$, this class $\xi_{\mathfrak{p}}$ gives the unique boundary point fixed by $f^{\prime}$. Conversely, if $h$ is an element of $\mathrm{Cr}_{2}(\mathbf{k})$ that fixes $\xi_{\mathfrak{p}}$, then $h$ determines an automorphism of $X$ preserving the fibration $\pi$; then, either $h$ is elliptic, or it is a Halphen twist. If $h$ is a Halphen twist, then some iterate of $h$ is in the group $P_{0}(X)$.

Example 4.8. Consider, over the field of complex numbers, an elliptic curve $E=\mathbf{C} / \Lambda$, and form the product $\mathbb{P}_{\mathbf{C}}^{1} \times E$. The involution $\sigma:([x: y], z) \mapsto([-x:$ $y],-z)$ of $\mathbb{P}_{\mathbf{C}}^{1} \times E$ preserves exactly two fibers $\{[0: 1]\} \times E$ and $\{[1: 0]\} \times E$, with 4 fixed points on each of them. Blowing up these 8 points, and then taking the quotient by (the lift of) $\sigma$, we get a rational surface $X_{E}$, with a genus 1 fibration $\pi: X_{E} \rightarrow \mathbb{P}_{\mathbf{C}}^{1}$, and exactly two singular fibers (each of type $\mathrm{I}_{0}^{*}$ in Kodaira's table). The multiplicative group acts by $([x: y], z) \mapsto([s x: y], z)$ on $\mathbb{P}_{\mathbf{C}}^{1} \times E$, hence also on $X_{E}$. So, the kernel of the homomorphism $\operatorname{Aut}\left(X_{E}\right) \mapsto$ $\mathrm{GL}\left(\mathrm{NS}\left(X_{E}\right)\right)$ contains a multiplicative group; in particular it is infinite. According to Theorem 4.7, there is no Halphen twist preserving the fibration $\pi: X_{E} \rightarrow \mathbb{P}_{\mathbf{C}}^{1}$. This can be seen directly on that example: a finite index subgroup of Aut $\left(X_{E}\right)$ preserves the 8 exceptional curves of self-intersection -2 contained in the fibers above $[0: 1]$ and $[1: 0]$, as well as the class of a general fiber; as a consequence, this finite index subgroup acts trivially on the subspace $\xi_{9}^{\perp} \subset \mathbf{Z}^{1,9}$, hence also on $\mathbf{Z}^{1,9}$. So, Aut $\left(X_{E}\right)$ cannot contain any Halphen twist.

Proof. The first assertion, i.e. the existence of an invariant Halphen pencil $\mathfrak{p}$ (up to conjugacy) is due to Gizatullin (see [19]): his proof works also in characteristic 2 or 3 , as noted in [9]. Blowing-up the base-points of $\mathfrak{p}$, one gets a Halphen surface $X$, and $f$ is conjugate to an automorphism $f_{X}$ of $X$; this automorphism preserves the fibration $\pi: X \rightarrow \mathbb{P}^{1}$ given by $\mathfrak{p}$. 
If $\pi$ were quasi-elliptic, $f_{X}$ would preserve the cusp curve $\Sigma_{X}$. Thus, $f_{X}$ would preserve the class $\left[\Sigma_{X}\right]$ of the curve of cusps, as well as the class $\xi_{X}$ of a general fiber. Since the intersection product $\left[\Sigma_{X}\right] \cdot \xi_{X}$ is positive, $f_{X}$ should preserve a class $\left[\Sigma_{X}\right]+m \xi_{X}$ with positive self-intersection, and $f_{X}$ should be elliptic, in contradiction with $f$ being a Halphen twist. Thus, $\pi$ is an elliptic fibration: its general fibers are curves of genus 1 .

Consider the kernel $K$ of the homomorphism Aut $(X) \rightarrow \mathrm{GL}(\mathrm{NS}(X))$. Since $\mathrm{Pic}^{0}(X)$ is trivial, $K$ is a linear algebraic group; we denote by $K^{0}$ the connected component of the identity in $K$. First, we show that $K$ is finite, or equivalently that $K^{0}$ is trivial. Recall from Lemma 4.5 that $\operatorname{Aut}(X)$ permutes the fibers of $\pi: X \rightarrow \mathbb{P}_{\mathbf{k}}^{1}:$ this provides an algebraic homomorphism $\rho: K \rightarrow \operatorname{Aut}\left(\mathbb{P}^{1}\right)$ such that $\pi \circ g=\rho(g) \circ \pi$ for all $g \in K$. The kernel of $\rho$ is a linear algebraic group, preserving each of the fibers of $\pi$. Since every algebraic homomorphism of a connected linear algebraic group to a curve of genus 1 is trivial, the kernel of $\rho$ is finite. The Euler characteristic of $X$ (using $\ell$-adic cohomology if $\operatorname{char}(\mathbf{k})>$ 0 ) is equal to 12 because $X$ is obtained from $\mathbb{P}_{\mathbf{k}}^{2}$ by a sequence of 9 successive blow-ups; hence, $\pi$ has at least one singular fiber (see [12], Proposition 5.1.6, p. 290). Since, the group $\rho\left(K^{0}\right) \subset \mathrm{PGL}_{2}(\mathbf{k})$ fixes all the critical values of $\pi$, it fixes at least one point; in particular, $\operatorname{dim}\left(K^{0}\right)=0,1$, or 2 . If $\operatorname{dim}\left(K^{0}\right)=1$, the (Zariski closures) of the orbits of $K^{0}$ form a pencil of rational curves, and this pencil is Aut $(X)$-invariant, because $K^{0}$ is a normal subgroup of $\operatorname{Aut}(X)$. Then, $f_{X}$ would preserve two pencils, the Halphen pencil and this pencil of rational curves, and $f$ would not be a Halphen twist: the two pencils would provide two fixed points of $f$ on the boundary of $\mathbb{H}_{\infty}$, and $f$ would not be a parabolic isometry of $\mathbb{H}_{\infty}$. This contradiction shows that $\operatorname{dim}\left(K^{0}\right) \neq 1$. If the dimension of $K^{0}$ is 2 , then $\rho\left(K^{0}\right)$ is the group of affine transformations $z \mapsto a z+b$ of the line; the subgroup of elements $g$ in $K^{0}$ such that $\rho(g)$ is a translation $z \mapsto z+b$ is invariant under the action of $\operatorname{Aut}(X)$ on $K^{0}$ by conjugacy, its dimension is equal to 1 , and we also get a contradiction. Thus, $K$ is finite.

Now, we show that $|K| \leq 8$ !. Consider the birational morphism $\tau: X \rightarrow$ $\mathbb{P}_{\mathbf{k}}^{2}$ given by (the inverse) of the blow-up of the base-points of the pencil $\mathfrak{p}$. Since $K$ acts trivially on $\mathrm{NS}(X)$, it preserves the classes of the 9 irreducible curves contracted by $\tau$, and since these curves have negative self-intersections it preserves each of these curves. Thus, there is a finite subgroup $K_{0}$ of Aut $\left(\mathbb{P}_{\mathbf{k}}^{2}\right)$ and an isomorphism $\varphi: K \rightarrow K_{0}$ such that $\varphi(g) \circ \tau=\tau \circ g$ for every $g \in K$. The group $K_{0}$ preserves $\mathfrak{p}$, permuting its members but fixing each of its base-points (including infinitely near ones). Conversely, the subgroup $G$ of $\operatorname{Aut}\left(\mathbb{P}_{\mathbf{k}}^{2}\right)$ that preserves each of these 9 points is an algebraic subgroup that lifts to a subgroup of $\operatorname{Aut}(X)$, and this subgroup acts trivially on $\mathrm{NS}(X)$ because it preserves the vectors $\mathbf{e}_{1}, \ldots, \mathbf{e}_{9}$ of the corresponding geometric basis as well as the vector 
$\mathbf{e}_{0}$ (since $G$ preserves the class of a line in $\mathbb{P}_{\mathbf{k}}^{2}$ ). Thus, $G$ coincides with $K_{0}$ and is finite. So, we just have to prove that $|G| \leq 8$ !. For that, we identify $\operatorname{Aut}\left(\mathbb{P}_{\mathbf{k}}^{2}\right)=\mathrm{PGL}_{3}(\mathbf{k})$ to the open subset of $\mathbb{P}_{\mathbf{k}}^{8}$ corresponding to the coefficients $\left[h_{i, j}\right]$ of matrices such that $\operatorname{det}\left(h_{i, j}\right) \neq 0$. The finite group $G$ is determined by 9 algebraic equations $h\left(q_{k}\right)=q_{k}$, where the $q_{k}$ are the base-points of $\mathfrak{p}$. Let us show that the degrees of these equations is bounded by 8 . Indeed, if $h=\left(h_{i, j}\right)$ is an element of $\mathrm{PGL}_{3}(\mathbf{k})$ and $q_{0}$ is a point of $\mathbb{P}_{\mathbf{k}}^{2}$, then the equation $h\left(q_{0}\right)=q_{0}$ is linear in the coefficients $h_{i, j}$. Then, if we blow-up $q_{0}$ and choose a point $q_{1}$ on the exceptional divisor, the equation $h\left(q_{1}\right)=q_{1}$ is linear in the coefficients of $D h_{q_{0}}$, and therefore also in the coefficients of $h$. Going on one step further, blowinq-up $q_{1}$ and choosing a point $q_{2}$ on the exceptional divisor, the equation $h\left(q_{2}\right)=q_{2}$ is a linear constraint on the second jet of $h$ at $q_{0}$; thus, it is a quadratic equation in the coefficients $\left[h_{i, j}\right]$ (3). If we do a tower of $k$ successive blow-ups, with $q_{i+1}$ infinitely near $q_{i}$, then the equation $h\left(q_{k}\right)=q_{k}$ has degree $\max (k-1,1)$ in the coefficients of $h$. Since, in our setting, we have 9 base-points, we get 9 equations of respective degrees $d_{1}, \ldots, d_{9}$ in the coefficients $\left[h_{i, j}\right]$. By the Bezout Theorem, the number of solutions of this system of equations, if finite, is at most $d_{1} \times \cdots \times d_{9}$. The worst case is for

$$
\left(d_{1}, d_{2}, d_{3}, \ldots, d_{9}\right)=(1,1,2, \ldots, 8)
$$

and gives the upper bound $8 !=40320$.

Example 4.9. Keep the notation of the proof. Given any Halphen pencil, the group $K$ can be described explicitly. We just give two examples. First, assume that the 9 base-points of the pencil $\mathfrak{p}$ contain a projective basis $\left(q_{0}, q_{1}, q_{2}, q_{3}\right)$ of $\mathbb{P}_{\mathbf{k}}^{2}$. Then $K$ is trivial. This corresponds to the generic situation.

Now, assume that the index $m$ of $\mathfrak{p}$ is larger than 1 and the unique multiple fiber $C$ of the fibration $\pi: X \rightarrow \mathbb{P}_{\mathbf{k}}^{1}$ is smooth. Then, the projection $C_{0}=\tau(C) \subset$ $\mathbb{P}_{\mathbf{k}}^{2}$ is a smooth cubic curve, and is invariant under $K_{0}$. Since $K_{0}$ fixes at least one point of $C_{0}$, it acts by group-automorphisms on $C_{0}$. Moreover, an element of $\operatorname{Aut}\left(\mathbb{P}_{\mathbf{k}}^{2}\right)$ which preserves $C_{0}$ pointwise is the identity. So, $\left|K_{0}\right|=|K|$ is bounded by 6 if $\operatorname{char}(\mathbf{k}) \neq 2,3$, by 12 if $\operatorname{char}(\mathbf{k})=3$, and by 24 if $\operatorname{char}(\mathbf{k})=2$.

Remark 4.10. It may be the case that $K$ is automatically trivial when $X$ supports a Halphen twist. We don't have any counter-example to this assertion.

3 To see it, assume $h$ fixes the point $[0: 0: 1]$, then in the affine coordinates $(x, y)$ we have

$$
h\left(\begin{array}{l}
x \\
y
\end{array}\right)=\left(\begin{array}{c}
\frac{a x+b y}{1+u x+v y} \\
\frac{c x+d y}{1+u x+v y}
\end{array}\right)=\left(\begin{array}{c}
(a x+b y)\left(1-(u x+v y)+(u x+v y)^{2}+\cdots\right) \\
(c x+d y)\left(1-(u x+v y)+(u x+v y)^{2}+\cdots\right)
\end{array}\right) .
$$

Thus, the coefficients of the jet of degree $d$ of $h$ at the origin $(0,0)$ are polynomial functions of degree $\leq d$ in the coefficients of $h$. 
Recall that $P_{0}(\mathfrak{p}) \subset \operatorname{Bir}\left(\mathbb{P}_{\mathbf{k}}^{2} ; \mathfrak{p}\right)$ is the subgroup of automorphisms $f \in \operatorname{Bir}\left(\mathbb{P}_{\mathbf{k}}^{2} ; \mathfrak{p}\right)$ whose image in $\mathrm{GL}(\mathrm{NS}(X))$ lies in the abelian group $P^{*} \subset \mathrm{O}\left(\mathbf{Z}^{1,9}\right)^{+}$given by Lemma 4.3 .

Corollary 4.11. Let $f$ be a Halphen twist, and let $\mathfrak{p}$ be the unique $f$-invariant Halphen pencil. The subgroup $P_{0}(\mathfrak{p}) \subset \operatorname{Bir}\left(\mathbb{P}_{\mathbf{k}}^{2} ; \mathfrak{p}\right)$ is an extension

$$
1 \rightarrow K \rightarrow P_{0}(\mathfrak{p}) \rightarrow \mathbf{Z}^{s} \rightarrow 0
$$

of an abelian group of rank $s \leq 8$ by a finite group $K$ with $|K| \leq B_{H} \leq 8$ !.

There is a normal subgroup $P(\mathfrak{p}) \triangleleft P_{0}(\mathfrak{p})$ of index $\leq s^{2|K|}((|K|+1)$ !) which is a free abelian group of rank $s$.

This subgroup $P(\mathfrak{p})$ of $\operatorname{Bir}\left(\mathbb{P}_{\mathbf{k}}^{2} ; \mathfrak{p}\right)$ will play an important role in the next sections.

Proof. The previous theorem shows the first assertion. To prove the second assertion, consider any exact sequence $1 \rightarrow K \rightarrow P_{0} \rightarrow \mathbf{Z}^{s} \rightarrow 0$ where $K$ is a finite group, and $|K| \leq D$ for some positive integer $D$. The group $P_{0}$ normalizes $K$, acting by conjugation on it. There is a subgroup $P_{1}$ of $P_{0}$ of index $\leq D$ ! that centralizes $K$ : every element of $P_{1}$ commutes to every element of $K$. Set $n=2|K|$; hence $|K|$ divides $n$ and $n(n+1) / 2$. Then, set $P=\left\{f^{n} \mid f \in P_{1}\right\}$. The first remark is that $P$ is a subgroup of $P_{1}$. Indeed, given any pair of elements $f$ and $g$ in $P_{1}$, we have $f g=g f c$ for some $c$ in $K$; then, for every $m \geq 1$, $f^{m} g=g f^{m} c^{m}$ and $f g^{m}=g^{m} f c^{m}$ because $c$ is central, and this gives $(f g)^{m}=$ $g^{m} f^{m} c^{1+2+3+\ldots+m}$; with $m=n$ we obtain that $f^{n} g^{n}=(f g)^{n}$ so that $P$ is stable under multiplication. The second remark is that $P \cap K=\{1\}$. The third remark is that the projection of $P$ in $\mathbf{Z}^{S}$ is an injective homomorphism, the image of which is a finite index subgroup of $n \mathbf{Z}^{S}$. Thus, $P$ is a free abelian subgroup of $P_{1}$ of rank $s$ and index $\leq s^{n} D$. Since $n=2|K|$ we see that the index of $P$ in $P_{0}$ is $\leq s^{2 D} D(D !) \leq s^{2 D}((D+1) !)$. Moreover, $P$ is a normal subgroup of $P_{0}$ (because $P_{1}$ is normal and $P$ is characteristic).

\section{DiSJONCTION OF HOROBALLS}

In this section, we prove Corollary 5.3 which says that horoballs associated to Halphen twists are disjoint. This is the first technical input to apply results of small cancellation and geometric group theory.

Consider a family $C$ of convex subsets of $\mathbb{H}_{\infty}$. Let $R$ be a positive real number. By definition, $C$ is $R$-separated if $\operatorname{dist}_{\mathbb{H}_{\infty}}\left(C, C^{\prime}\right)>R$ for all pairs of distinct elements $C, C^{\prime} \in C$. A vector $v \in \mathbf{Z}^{1, \infty}$ is primitive if it is not a non-trivial multiple of some vector $v^{\prime} \in \mathbf{Z}^{1, \infty}$. 
Theorem 5.1. Let $\mathcal{P}$ be the set of integral, isotropic and primitive vectors $v$ in $\mathbf{Z}^{1, \infty}$ such that $v \cdot \mathbf{e}_{0}>0$. If $0<\varepsilon<1 / \sqrt{2}$, the family of horoballs $\left\{H_{v}(\varepsilon)\right\}_{v \in \mathcal{P}}$ is $\operatorname{arcosh}\left(\frac{1}{4 \varepsilon^{2}}\right)$-separated.

Remark 5.2. The set of horoballs $\left\{H_{v}(\varepsilon)\right\}_{v \in \mathcal{P}}$ is invariant by the group of isometries of $\mathbb{H}_{\infty}$ preserving integral points.

Proof. Consider two different horoballs $H_{v}(\varepsilon)$ and $H_{v^{\prime}}(\varepsilon)$ of $\mathcal{P}$. Consider $x \in$ $\left[v, v^{\prime}\right] \cap \partial H_{v}(\varepsilon)$ and $y \in\left[v, v^{\prime}\right] \cap \partial H_{v^{\prime}}(\varepsilon)$, where $\left[v, v^{\prime}\right]$ is the geodesic from $v$ to $v^{\prime}$. Set $m=v \cdot v^{\prime}$ (this is a positive integer), and write $x=\alpha v+\alpha^{\prime} v^{\prime}$ and $y=$ $\beta v+\beta^{\prime} v^{\prime}$. The points $x$ and $y$ belong to $\mathbb{H}_{\infty}$ so $x \cdot x=y \cdot y=1$, and by definition of the horospheres $x \cdot v=y \cdot v^{\prime}=\varepsilon$. This gives

$$
\begin{aligned}
& 2 \alpha \alpha^{\prime} m=2 \beta \beta^{\prime} m=1, \\
& \alpha^{\prime} m=\beta m=\varepsilon .
\end{aligned}
$$

Using these equalities we get:

$$
x \cdot y=\left(\alpha \beta^{\prime}+\alpha^{\prime} \beta\right) m=\frac{m}{4 \varepsilon^{2}}+\frac{\varepsilon^{2}}{m} .
$$

Since $m \geq 1$, we obtain:

$$
x \cdot y>\frac{1}{4 \varepsilon^{2}} .
$$

For $4 \varepsilon^{2}>1$ the two horoballs overlap, for $4 \varepsilon^{2}=1$ they are tangent at $x=y$, and for $4 \varepsilon^{2}<1$ they are disjoint: The distance between $H_{v}(\varepsilon)$ and $H_{v^{\prime}}(\varepsilon)$ is the distance between $x$ and $y$ and is equal to

$$
\operatorname{dist}_{\mathbb{H}_{\infty}}\left(H_{v}(\varepsilon), H_{v^{\prime}}(\varepsilon)\right)=\operatorname{dist}_{\mathbb{H}_{\infty}}(x, y) \geq \operatorname{arcosh}\left(\frac{1}{4 \varepsilon^{2}}\right) .
$$

This concludes the proof.

Let $\mathfrak{p}$ be a Halphen pencil. Recall from section Section 4.3 that $\xi_{\mathfrak{p}}$ denotes the anticanonical class of the surface obtained by blowing up the nine basepoints of $\mathfrak{p}$. Since $\xi_{\mathfrak{p}} \in Z$ is primitive and isotropic, and the Cremona group permutes the set of primitive, isotropic vectors, Theorem 5.1 provides the following result.

Corollary 5.3. Consider the set of all horoballs of the form

$$
f\left(H_{\xi_{\mathfrak{p}}}(\varepsilon)\right)=H_{f\left(\xi_{\mathfrak{p}}\right)}(\varepsilon)
$$

where $\mathfrak{p}$ is any Halphen pencil and $f$ is any element of $\mathrm{Cr}_{2}(\mathbf{k})$. Then for $0<$ $\varepsilon<1 / \sqrt{2}$, this set of horoballs is $\operatorname{arcosh}\left(\frac{1}{4 \varepsilon^{2}}\right)$-separated.

\section{GEOMETRIC GROUP THEORY AND CONCLUSION}

To prove Theorem B, we rely on the work of François Dahmani, Vincent Guirardel and Denis Osin (see [14]). 
6.1. Hyperbolic spaces and rotating families. First we recall one of the main results of [14].

Consider a geodesic metric space $X$ and a non-negative constant $\sigma$. A subset $Q$ of $X$ is $\sigma$-strongly quasiconvex if for any two points $x, y$ of $Q$ there exist $x^{\prime}, y^{\prime}$ in $Q$ and geodesics $\left[x^{\prime}, y^{\prime}\right],\left[x^{\prime}, x\right]$ and $\left[y, y^{\prime}\right]$ included in $Q$ such that $\max \left\{\operatorname{dist}\left(x, x^{\prime}\right), \operatorname{dist}\left(y, y^{\prime}\right)\right\} \leq \sigma$.

Let $\delta$ be a non-negative constant. A geodesic metric space $X$ is $\delta$-hyperbolic if every triangle in $X$ is $\delta$-thin, meaning that each side of the triangle is included in the $\delta$-neighborhood of the union of the two remaining sides. We shall say that $X$ is Gromov-hyperbolic if it is $\delta$-hyperbolic for some $\delta>0$.

Let $G$ be a group acting by isometries on a $\delta$-hyperbolic space $X$, for some $\delta>0$. Consider a $G$-invariant subset $C \subset X$, which we call the set of apices. For each $c \in C$, consider $G_{c} \subset \operatorname{Stab}(c)$ a subgroup of the stabilizer of $c$ in $G$, called the rotation subgroup. Following [14, Definition 5.1], we say that the family $\left(C,\left\{G_{c}\right\}_{c \in C}\right)$ is a very rotating family if it satisfies the following conditions:

(1) for every $g \in G$, for every $c \in C, G_{g c}=g G_{c} g^{-1}$,

(2) for every $c \in C$, for every $g \in G_{c} \backslash\{1\}$, and for every $x, y \in X$ satisfying $\operatorname{dist}(x, c) \in[20 \delta, 40 \delta], \operatorname{dist}(y, c) \in[20 \delta, 40 \delta]$, and $\operatorname{dist}(g x, y) \leq 15 \delta$,

any geodesic between $x$ and $y$ contains $c$.

Moreover, as in Section 5, the family is $\rho$-separated, for some $\rho>0$, if the distance between distinct apices is always strictly bigger than $\rho$.

Theorem 6.1 ([14, Theorem 5.3]). Let $G$ be a group acting by isometries on a $\delta$-hyperbolic geodesic space $X$. Let $\left(C,\left\{G_{c}\right\}_{c \in C}\right)$ be a $\rho$-separated very rotating family, for some $\rho>200 \delta$. Then the normal subgroup $N_{G}$ of $G$ generated by the groups $\left\{G_{c}\right\}_{c \in C}$ is a free product of a (usually infinite) subfamily of $\left\{G_{c}\right\}_{c \in C}$. Moreover, for every $c \in C, N_{G} \cap \operatorname{Stab}(c)=G_{c}$.

Remark 6.2. If $G_{c}$ is a proper subgroup of $\operatorname{Stab}(c)$, or if $G_{c}$ is abelian and $G$ has no non-trivial homomorphism onto an abelian group, then the normal subgroup generated by the groups $\left\{G_{c}\right\}_{c \in C}$ is a proper subgroup of $G$. In particular, if $f \in G_{c} \backslash\{\mathrm{Id}\}$, the smallest normal subgroup of $G$ containing $f$ is proper and non-trivial. See [14] for other consequences.

6.2. Cone-off construction. In our case, we shall apply Theorem 6.1 to $G=$ $\mathrm{Cr}_{2}(\mathbf{k})$, and the role of $G_{c}$ will be played by the subgroup $P(\mathfrak{p})$ of $\operatorname{Bir}\left(\mathbb{P}_{\mathbf{k}}^{2}, \mathfrak{p}\right)$. This group fixes a point $\xi_{\mathfrak{p}} \in \partial \mathbb{H}_{\infty}$, but has no fixed point inside $\mathbb{H}_{\infty}$. We shall modify $\mathbb{H}_{\infty}$ to bring this fixed point inside the space: this is done in Section 6.2.2. Then, we need to modify this cone-off, because if we go deeply inside the horoballs the very rotating property (2) above will fail. For that, we dig a hole 
in the cones, and this is explained in Section 6.2.3. For more details on this cone-off constructions, see [14, Sections 5.3 and 7.1] or [13, Section 3].

6.2.1. Hyperbolic cones (see [6]). Consider a metric space $Y$ and a real number $r_{0}>0$. The hyperbolic cone over $Y$ of height $r_{0}$, denoted by Cone $\left(Y, r_{0}\right)$, is the quotient of $Y \times\left[0, r_{0}\right]$ by the equivalence relation:

$$
\left(y_{1}, r_{1}\right) \sim\left(y_{2}, r_{2}\right) \text { if and only if } r_{1}=r_{2}=0 \text { or }\left(y_{1}, r_{1}\right)=\left(y_{2}, r_{2}\right) .
$$

By definition, the point $(y, 0)$ is the apex of $\operatorname{Cone}\left(Y, r_{0}\right)$. The metric on this cone is defined by the formula $\operatorname{dist}_{\text {Cone }\left(Y, r_{0}\right)}\left((y, r),\left(y^{\prime}, r^{\prime}\right)\right)=\operatorname{arcosh}\left(\cosh r \cosh r^{\prime}-\sinh r \sinh r^{\prime} \cos \theta\left(y, y^{\prime}\right)\right)$, where $\theta\left(y, y^{\prime}\right)=\min \left(\pi, \frac{\operatorname{dist}_{Y}\left(y, y^{\prime}\right)}{\sinh r_{0}}\right)$. By [6, Chap. I.5 Prop 5.10], Cone $\left(Y, r_{0}\right)$ is a geodesic metric space if and only if $Y$ is geodesic.

6.2.2. Cone-off. Consider a metric space $X$, a family $Y=\left\{Y_{i}\right\}_{i}$ of subsets of $X$, and $r_{0}$ a positive constant. The cone-off of $Y$ over $X$, denoted by $C\left(X, Y, r_{0}\right)$, is the space obtained from the disjoint union of $X$ and of the cones $\operatorname{Cone}\left(Y_{i}, r_{0}\right)$, for all $i$, by gluing each subspace $Y_{i}$ in $X$ to $Y_{i} \times\left\{r_{0}\right\}$ in $\operatorname{Cone}\left(Y_{i}, r_{0}\right)$ via the identification

$$
y \in Y_{i} \mapsto\left(y, r_{0}\right) \in \operatorname{Cone}\left(Y_{i}, r_{0}\right)
$$

By [13, Proposition 2.1.5], for $\left(y, r_{0}\right)$ and $\left(y^{\prime}, r_{0}\right) \in \operatorname{Cone}\left(Y_{i}, r_{0}\right)$ we have

$$
\operatorname{dist}_{\text {Cone }\left(Y_{i}, r_{0}\right)}\left(\left(y, r_{0}\right),\left(y^{\prime}, r_{0}\right)\right) \leq \operatorname{dist}_{X}\left(y, y^{\prime}\right) .
$$

Let us construct a metric on $C\left(X, Y, r_{0}\right)$. Given $x$ and $x^{\prime}$ in $C\left(X, Y, r_{0}\right)$, set

$\left\|x-x^{\prime}\right\|= \begin{cases}\operatorname{dist}_{X}\left(x, x^{\prime}\right) & \text { if } x, x^{\prime} \in X \text { and there is no } i \text { such that } x, x^{\prime} \in Y_{i} \\ \operatorname{dist}_{\operatorname{Cone}\left(Y_{i}, r_{0}\right)}\left(x, x^{\prime}\right) & \text { if there exists } Y_{i} \text { such that } x, x^{\prime} \in \operatorname{Cone}\left(Y_{i}, r_{0}\right) \\ +\infty & \text { otherwise. }\end{cases}$

Then, consider a chain $C$ between $x$ and $x^{\prime}$, i.e. a finite sequence of points $x=$ $z_{1}, z_{2}, \ldots, z_{n}=x^{\prime}$ in $C\left(X, Y, r_{0}\right)$. Define its length to be $\ell(C)=\sum_{i=1}^{n-1}\left\|z_{i+1}-z_{i}\right\|$. By [13, Proposition 3.1.7], a distance is defined on the cone-off as follows:

$$
\operatorname{dist}_{C\left(X, Y, r_{0}\right)}=\inf \left\{\ell(C) \mid C \text { is a chain between } x \text { and } x^{\prime}\right\} .
$$

The following theorem concerns metric graphs with isometric edges (edges of the same length); this ensures that the cone-off is geodesic ([6, Theorem I.7.19]). As in [14], page 77, set

$$
\delta_{U}=900, \text { and } r_{U}=5 \times 10^{12} .
$$


Theorem 6.3. Given any $r_{0} \geq r_{U}$, there exists $\delta_{c}>0$ such that : if $X$ is a $\delta_{c}$-hyperbolic metric graph with pairwise isometric edges, and if $Q$ is a $40 \delta_{c^{-}}$ separated family of $10 \delta_{c}$-strongly quasiconvex subsets of $X$, then the cone-off $C\left(X, Q, r_{0}\right)$ is globally $\delta_{U}$-hyperbolic.

Proof. This statement follows from Corollary 5.39 of [14]. Indeed, with the notation from [14], the fellow traveling constant of the family $Q$ satisfies $\Delta(Q)=0$ because $Q$ is $40 \delta_{c}$-separated. Hence, all hypotheses of Theorem 5.38 of [14] are satisfied.

6.2.3. Parabolic cone-off (see [14, §7.1]). For any $Q \in Q$, we consider the hyperbolic cone Cone $\left(Q, r_{0}\right)$. For any $p, q \in \partial Q$ with $\operatorname{dist}_{X}(p, q) \leq \pi \sinh \left(r_{0}\right)$, and for any geodesic $[p, q]$ in $\operatorname{Cone}\left(Q, r_{0}\right)$, we consider the filled triangle $T_{[p, q]} \subset$ Cone $\left(Q, r_{0}\right)$ bounded by the geodesics $[p, q],\left[p, c_{Q}\right]$ and $\left[q, c_{Q}\right]$; this triangle is a cone over $[p, q]$. For any $Q \in Q$, we define

$$
B_{Q}=\bigcup T_{[p, q]}
$$

where the union runs over all geodesics $[p, q]$ in $\operatorname{Cone}\left(Q, r_{0}\right)$ of length $\leq \pi \sinh \left(r_{0}\right)$ and with endpoints in $\partial Q$.

Definition 6.4. Consider $X$ a Gromov-hyperbolic space and $Q$ a $R$-separated family of convex subsets. The parabolic cone-off $C^{\prime}\left(X, Q, r_{0}\right)$ is the subset of $C\left(X, Q, r_{0}\right)$ given by:

$$
\left(C\left(X, Q, r_{0}\right) \backslash \bigcup_{Q \in Q} \operatorname{Cone}\left(Q, r_{0}\right)\right) \cup\left(\bigcup_{Q \in Q} B_{Q}\right) .
$$

It is endowed with the induced path metric.

In the following lemma, $r_{0} \geq r_{U}$ and $\delta_{c}$ is given by Theorem 6.3. For the definition of horoballs in a Gromov-hyperbolic graph see [14, Section 7.1 p.111].

Lemma 6.5. [14, Lemma 7.4] Let $X$ be a $\delta_{c}$-hyperbolic graph with isometric edges and $\mathcal{H}$ a $40 \delta_{c}$-separated system of horoballs. Then the parabolic coneoff $C^{\prime}\left(X, Q, r_{0}\right)$ is $16 \delta_{U}$-hyperbolic.

The following Lemma is proved in [14]. Although the statement is given in the context of relatively hyperbolic group, its proof just needs a separated set of horoballs.

Lemma 6.6. [14, Lemma 7.5] Let $G$ be a group acting isometrically on a $\delta_{c^{-}}$ hyperbolic graph $X$ with isometric edges. Let $H_{i} \subset X, i \in I$, be a family of horoballs indexed by a set I. For each index $i$, let $G\left(H_{i}\right) \subset G$ be the stabilizer of $H_{i}$ and $N_{i} \triangleleft G(H)$ be a normal subgroup of $G\left(H_{i}\right)$. Assume that

(i) the $H_{i}$ are in pairwise disjoint orbits under the action of $G$; 
(ii) the set of distinct horoballs

$$
\mathcal{H}=\left\{g\left(H_{i}\right) \mid i \in I, g \in G / G\left(H_{i}\right)\right\}
$$

is $40 \delta_{c}$-separated.

(iii) $\operatorname{dist}_{X}(x, g x) \geq 4 \pi \sinh \left(r_{0}\right)$ for each $g \in N_{i} \backslash\{1\}$ and each $x \in \partial H_{i}$.

Then the family of all $G$-conjugates of the $N_{i}$ defines a $2 r_{0}$-separated very rotating family on $C^{\prime}\left(X, \mathcal{H}, r_{0}\right)$.

6.3. Conclusion. We can now prove a strong form of Theorem B.

Theorem B'. There exists a positive integer $n_{0}$ with the following property. Let $\mathbf{k}$ be a field. Consider the collection of all iterates $g^{n_{0}}$ of all Halphen twists $g \in \mathrm{Cr}_{2}(\mathbf{k})$. Then the normal subgroup generated by this collection is a nontrivial proper subgroup of $\mathrm{Cr}_{2}(\mathbf{k})$.

Moreover, this normal subgroup is a free product of free abelian groups of rank $\leq 8$.

Proof of Theorem B'. First, assume that the field $\mathbf{k}$ is algebraically closed.

Consider the action of $\mathrm{Cr}_{2}(\mathbf{k})$ on $\mathbb{H}_{\infty}$. In order to apply the results of [14], which are stated in the case of Gromov-hyperbolic graphs, we need the following classical construction. Let $\mathcal{G}$ be the metric graph defined as follows. The vertices of $\mathcal{G}$ are the points of $\mathbb{H}_{\infty}$ and we put an edge between two vertices $x$ and $y$ if their distance in $\mathbb{H}_{\infty}$ is at most 1 . This graph is endowed with the path metric for which each edge is isometric to the interval $[0,1]$. The Cremona group acts on $\mathcal{G}$ by isometries. We denote by $i: \mathbb{H}_{\infty} \hookrightarrow \mathcal{G}$ the inclusion. It is a $(1,1)$-quasi-isometry:

$$
\operatorname{dist}_{\mathcal{G}}(i(x), i(y))-1 \leq \operatorname{dist}_{\mathbb{H}_{\infty}}(x, y) \leq \operatorname{dist}_{\mathcal{G}}(i(x), i(y))
$$

for any $x, y \in \mathbb{H}_{\infty}$. Then $\mathcal{G}$ is $\delta_{\mathcal{G}}$-hyperbolic with $\delta_{\mathcal{G}}=\ln (1+\sqrt{2})+1$.

Fix $\frac{1}{\sqrt{2}}>\varepsilon>0$ such that $\operatorname{arcosh}\left(\frac{1}{4 \varepsilon^{2}}\right) \geq 40 \delta_{\mathcal{G}}$. For every Halphen pencil $\mathfrak{p}$, consider the horoball $H_{\mathfrak{p}}(\varepsilon)$ in $\mathbb{H}_{\infty}$. By Corollary 5.3, the family of translates of all the $H_{\mathfrak{p}}(\varepsilon)$ under $\mathrm{Cr}_{2}(\mathbf{k})$ is $R_{\mathcal{\varepsilon}}$-separated for $R_{\mathcal{\varepsilon}}=\operatorname{arcosh}\left(\frac{1}{4 \mathcal{E}^{2}}\right) \geq 40 \delta_{\mathcal{G}}$. To each horoball $H_{\mathfrak{p}}(\varepsilon)$ of $\mathbb{H}_{\infty}$, we associate the subgraph $H_{\mathfrak{p}}$ of $\mathcal{G}$ spanned by the vertices $\left\{i(x) \mid x \in H_{\mathfrak{p}}(\varepsilon)\right\}$. This is a 0 -strongly quasiconvex subgraph of $\mathcal{G}$ and the family of all translates $g H_{\mathfrak{p}}$ (for $g$ in $\mathrm{Cr}_{2}(\mathbf{k})$ and $\mathfrak{p}$ a Halphen pencil) is again $R_{\mathcal{E}}$-separated.

Set $r_{0}=r_{U}$. With $\lambda=\delta_{c} / \delta_{\mathcal{G}}$, the scaled graph $\lambda \mathcal{G}$ (in which each edge has length $\lambda$ ) is $\delta_{c}$-hyperbolic. We call it $\mathcal{G}^{\prime}$, and we denote by $H_{\mathfrak{p}}^{\prime} \subset \mathcal{G}^{\prime}$ and $\partial H_{\mathfrak{p}}^{\prime} \subset \mathcal{G}^{\prime}$ the subsets of $\mathcal{G}^{\prime}$ corresponding to $i\left(H_{\mathfrak{p}}\right), i\left(\partial H_{\mathfrak{p}}\right) \subset \mathcal{G}$. Now the set $\mathcal{H}^{\prime}$ of translates of $H_{\mathfrak{p}}^{\prime}$ under $\mathrm{Cr}_{2}(\mathbf{k})$, for all Halphen pencils invariant by some Halphen twists, is $\lambda R_{\varepsilon}$-separated; the choice for $\varepsilon$ implies that it is 
$40 \delta_{c}$-separated. By Lemma 6.5, the parabolic cone-off $C^{\prime}\left(\mathcal{G}^{\prime}, \mathcal{H}^{\prime}, r_{0}\right)$ is $16 \delta_{U^{-}}$ hyperbolic.

Let $\operatorname{Bir}\left(\mathbb{P}_{\mathbf{k}}^{2} ; \mathfrak{p}\right)$ be the stabilizer of $\mathfrak{p}$ in $\mathrm{Cr}_{2}(\mathbf{k})$, and let $P(\mathfrak{p}) \triangleleft P$ be the normal free abelian subgroup given by Corollary 4.11, Using Lemma 4.6, we find an integer $n$, that does not depend on $\mathfrak{p}$, such that

$$
\operatorname{dist}_{\mathcal{G}^{\prime}}\left(x, g^{n} x\right) \geq 4 \pi \sinh \left(r_{0}\right)
$$

for any $g \in P(\mathfrak{p}) \backslash\{\mathrm{Id}\}$ and for any $x \in \partial H_{\mathfrak{p}}{ }^{\prime}(\varepsilon)$. Then, we set $N_{\mathfrak{p}}=\left\{g^{n} \mid g \in\right.$ $P(\mathfrak{p})\}$. This is a subgroup because $P(\mathfrak{p})$ is abelian, and it is clearly normal in $\operatorname{Bir}\left(\mathbb{P}_{\mathbf{k}}^{2} ; \mathfrak{p}\right)$. Moreover, the Corollary 4.11 shows that there is a uniform integer

$$
n_{0}=n \times\left(8^{2 B_{H}} \times\left(B_{H}+1\right) !\right) !
$$

such that $g^{n_{0}}$ is in some $N_{\mathfrak{p}}$ for every Halphen twist $g$.

The family of horoballs $H_{\mathfrak{p}}^{\prime}$ and normal subgroups $N_{\mathfrak{p}} \subset \operatorname{Bir}\left(\mathbb{P}_{\mathbf{k}}^{2} ; \mathfrak{p}\right)$ satisfies the hypotheses of Lemma 6.6, so the family of $\mathrm{Cr}_{2}(\mathbf{k})$-conjugates of the $N_{\mathfrak{p}}$ defines a $2 r_{0}$-separated very rotating family on $C^{\prime}\left(\mathcal{G}^{\prime}, \mathcal{H}^{\prime}, r_{0}\right)$. By hypothesis on $r_{0}=r_{U}=10^{6} \delta_{U}, 2 r_{0}>200 \times\left(16 \delta_{U}\right)$ (see Equation (6.5)). Applying Theorem 6.1, we get the expected result.

If the field $\mathbf{k}$ is not algebraically closed, the first step is to replace it by an algebraic closure $\mathbf{k}^{\prime}$, so as to apply Theorem 6.1. The normal subgroup $F$ of $\mathrm{Cr}_{2}\left(\mathbf{k}^{\prime}\right)$ generated by the iterates $g^{n_{0}}$ for all Halphen twists $g \in \mathrm{Cr}_{2}(\mathbf{k})$ is a free product of abelian groups. Since $\mathrm{PGL}_{3}(\mathbf{k})$ does not embed into such a group, the intersection $F \cap \mathrm{Cr}_{2}(\mathbf{k})$ is a proper normal subgroup of $\mathrm{Cr}_{2}(\mathbf{k})$.

To conclude, we need to show that $\mathrm{Cr}_{2}(\mathbf{k})$ always contains Halphen twists: this is done in the examples below.

\subsection{Three examples.}

6.4.1. Halphen twists. Let $\mathbf{k}$ be a field of characteristic $p \neq 2$. Let $C \subset \mathbb{P}_{\mathbf{k}}^{2}$ be a smooth cubic curve defined over $\mathbf{k}$, together with a point $q \in C(\mathbf{k})$. By definition, the Jonquières involution $s_{q}$ is a birational involution of the plane that preserves the pencil of lines through the point $q$ and fixes $C$ pointwise. If $L$ is a line containing $q$, it intersects $C$ on a pair of points (except if the line is tangent to $C$ ) and the restriction $s_{q \mid L}$ is the unique involution fixing these two points (this involution does not exist if $\operatorname{char}(\mathbf{k})=2$ ). This involution is defined over $\mathbf{k}$. If one blows up the point $q$ and the points of tangency of the 4 lines containing $q$ which are tangent to $C$, the involution $s_{q}$ lifts to a regular involution of a rational surface $X_{q} \rightarrow \mathbb{P}_{\mathbf{k}}^{2}$.

Now, if we start with two points $q$ and $q^{\prime}$ in $C(\mathbf{k})$, we can lift $s_{q}$ and $s_{q^{\prime}}$ simultaneously as a pair of automorphisms $s_{Y}$ and $s_{Y}^{\prime}$ of a rational surface $Y$ ( $Y$ is the blow-up of the plane in 10 points that dominates $X_{q}$ and $X_{q^{\prime}}$ ). Jérémy Blanc proves in [1] that 
- there are no relations between $s_{Y}$ and $s_{Y}^{\prime}$ : the group they generate is isomorphic to $\mathbf{Z} / 2 \mathbf{Z} \star \mathbf{Z} / 2 \mathbf{Z}$.

- the composition $s_{Y} \circ s_{Y}^{\prime}$ is a parabolic automorphism (hence a Halphen twist).

Thus, to construct examples of Halphen twists in characteristic $\neq 2$, we only need to construct a smooth cubic curve and two points $q, q^{\prime}$ of $C(\mathbf{k})$. If $\operatorname{char}(\mathbf{k}) \neq 7$, one can take the curve defined by $x^{3}+y^{3}+z^{3}+x y z=0$, with $q=[1:-1: 0]$ and $q^{\prime}=[1: 0:-1]$. If $\operatorname{char}(\mathbf{k})=7$, one can take the curve $y^{2} z=x^{3}+z^{3}$, with $q=[-1: 0: 1]$ and $q^{\prime}=[0: 1: 0]$.

6.4.2. Characteristic 2. Let us describe another strategy that works in characteristic 2. Consider a field $\mathbf{k}$ of characteristic 2 . In $\mathbb{P}^{1} \times \mathbb{P}^{2}$, with affine coordinates $(t) \times(x, y)$, consider the surface $X \subset \mathbb{P}^{1} \times \mathbb{P}^{2}$ given by the equation

$$
y^{2}+x y+t^{3} y=x^{3}+1 .
$$

Let $\pi: X \rightarrow \mathbb{P}^{1}$ be the first projection. For $t \neq \infty$, the fiber $F_{t}=\pi^{-1}(t)$ is a cubic curve in Weierstrass form. There are two sections of $\pi$, given by $t \mapsto(-1,0)=$ $[-1: 0: 1]$ and $t \mapsto[0: 1: 0]$. Let $f: X \rightarrow X$ be the birational transformation of $X$ that preserves each of the fibers of $\pi$ and translates the first section to the second. In [25], William E. Lang shows that this Weierstrass pencil is a Halphen pencil with twelve singular irreducible curves. Thus, there is no -2 curve on $X$, and this implies that $f$ is a Halphen twist (see [9], Theorem 2.10).

6.4.3. An example over $\mathbf{C}$. Our last example shows that $n_{0}$ must be larger than 1 in Theorem B and B'.

Proposition 6.7. There is a Halphen twist $f$ in $\mathrm{Cr}_{2}(\mathbf{C})$ such that

(1) $f$ preserves every member of its invariant pencil and

(2) the smallest normal subgroup of $\mathrm{Cr}_{2}(\mathbf{C})$ that contains $f$ is equal to $\mathrm{Cr}_{2}(\mathbf{C})$.

Note that (1) implies that $f$ acts as a translation on the general member of its invariant pencil.

Proof. Consider the ring of Eisenstein integers $\Lambda:=\mathbf{Z}[\mathrm{j}] \subset \mathbf{C}$, where j a primitive cubic root of unity. Denote by $E$ the elliptic curve $\mathbf{C} / \Lambda$ and by $A$ the abelian surface $E \times E$. The two matrices

$$
F=\left(\begin{array}{ll}
1 & 1 \\
0 & 1
\end{array}\right) \text { and } G=\left(\begin{array}{ll}
1 & 0 \\
1 & 1
\end{array}\right)
$$

generate $\mathrm{SL}_{2}(\mathbf{Z}) \subset \mathrm{GL}_{2}(\Lambda)$, and in particular the order 3 element

$$
U=\left(\begin{array}{ll}
0 & -1 \\
1 & -1
\end{array}\right) .
$$


Denote by $\Pi_{F}$ and $\Pi_{G}$ the fibration $A \rightarrow E$ given by the projection onto the second and the first factors. Since $\mathrm{GL}_{2}(\Lambda)$ preserves the lattice $\Lambda \times \Lambda$ of $\mathbf{C}^{2}$, the group $\mathrm{GL}_{2}(\Lambda)$ embeds into the group of automorphisms of $A$. The automorphism $F$ (resp. $G$ ) is a Halphen twist with respect to the fibration $\Pi_{F}$ (resp. $\left.\Pi_{G}\right)(\sqrt[4]{4})$. Taking the quotient of $A$ by $J(x, y)=(j x, j y)$ (an element of the center of $\mathrm{GL}_{2}(\Lambda)$ ), we get a (singular) surface $X_{0}$ on which $\mathrm{PGL}_{2}(\Lambda)$ acts faithfully. The surface $X_{0}$ is a rational surface: there is a birational map $\varphi: X_{0} \rightarrow \mathbb{P}_{\mathbf{C}}^{2}$ (see [22, Lemma 4.1]).

The automorphisms $F, G$, and $U$ determine automorphisms $f_{0}, g_{0}$, and $u_{0}$ of $X_{0}$; after conjugacy by $\varphi$, we get three elements $f, g, u$ of $\mathrm{Cr}_{2}(\mathbf{C})$ such that:

(1) $f$ and $g$ are Halphen twists (with respect to distinct pencils);

(2) $u$ has order 3 , and is contained in the group generated by $f$ and $g$.

Moreover, the fixed point set of $U$ is a finite subset of $A$, hence the fixed point set of $u_{0}$ is finite. The singularities of $X_{0}$ are quotient singularities, and their resolution only creates new rational curves. So, the fixed point set of $u$ does not contain any irrational curve, and this implies that $u$ is conjugate to an element of $\mathrm{PGL}_{3}(\mathbf{C})$ (see [2]). By Lemma 3.1 the smallest normal subgroup generated by $f$ and $g$ coincides with $\mathrm{Cr}_{2}(\mathbf{C})$.

Also, the linear map $(x, y) \mapsto(y, x)$ conjugates $F$ to $G$. So, the smallest normal subgroup generated by $f$ contains $g$, and the proposition is proven.

\section{REFERENCES}

[1] Jérémy Blanc. On the inertia group of elliptic curves in the cremona group of the plane. Michigan Math. J., 56(2):315-330, 2008.

[2] Jérémy Blanc. Linearisation of finite abelian subgroups of the Cremona group of the plane. Groups Geom. Dyn., 3(2):215-266, 2009.

[3] Jérémy Blanc. Elements and cyclic subgroups of finite order of the Cremona group. Comment. Math. Helv., 86(2):469-497, 2011.

[4] Jérémy Blanc and Julie Déserti. Degree growth of birational maps of the plane. Ann. Sc. Norm. Super. Pisa Cl. Sci. (5), 14(2):507-533, 2015.

[5] E. Bombieri and D. Mumford. Enriques' classification of surfaces in char. p. III. Invent. Math., 35:197-232, 1976.

[6] Martin R. Bridson and André Haefliger. Metric spaces of non-positive curvature, volume 319 of Grundlehren der Mathematischen Wissenschaften [Fundamental Principles of Mathematical Sciences]. Springer-Verlag, Berlin, 1999.

[7] Serge Cantat. Sur les groupes de transformations birationnelles des surfaces. Annals of Mathematics. Second Series, 174:299-340, 2011.

[8] Serge Cantat. The Cremona groups. À paraître dans Proceedings of 2015 Summer Institute on Algebraic Geometry, AMS Proceedings of Symposia in Pure Mathematics, 2017.

\footnotetext{
${ }^{4}$ See [8] for Halphen twists on any surface. Here, this corresponds to the following equivalent facts: (a) the action of $F$ on the cohomology group $H^{1,1}(A ; \mathbf{R})$ satisfies $\left\|\left(F^{n}\right)^{*}\right\| \simeq \alpha n^{2}$ for some $\alpha>0$, or (b) the action of $F$ on the Neron-Severi group $\operatorname{NS}(A)$ satisfies $\left\|\left(F^{n}\right)^{*}\right\| \simeq \alpha n^{2}$, or (c) given any polarization $H$ on $A$, the degree $H \cdot\left(F^{n}\right)^{*} H$ grows like $\alpha n^{2}$.
} 
[9] Serge Cantat and Igor Dolgachev. Rational surfaces with a large group of automorphisms. J. Amer. Math. Soc., 25(3):863-905, 2012.

[10] Serge Cantat and Stéphane Lamy. Normal subgroups in the Cremona group. Acta Math., 210:31-94, 2013.

[11] Dominique Cerveau and Julie Déserti. Transformations birationnelles de petit degré, volume 19 of Cours Spécialisés [Specialized Courses]. Société Mathématique de France, Paris, 2013.

[12] François R. Cossec and Igor V. Dolgachev. Enriques surfaces. I, volume 76 of Progress in Mathematics. Birkhäuser Boston, Inc., Boston, MA, 1989.

[13] Rémi Coulon. Asphericity and small cancellation theory for rotation families of groups. Groups Geom. Dyn., 5(4):729-765, 2011.

[14] F. Dahmani, V. Guirardel, and D. Osin. Hyperbolically embedded subgroups and rotating families in groups acting on hyperbolic spaces. Mem. Amer. Math. Soc., 245(1156):v+152, 2017.

[15] Jeffrey Diller and Charles Favre. Dynamics of bimeromorphic maps of surfaces. Amer. J. Math., 123(6):1135-1169, 2001.

[16] Igor V. Dolgachev. Rational surfaces with a pencil of elliptic curves (russian). Izv. Akad. Nauk SSSR Ser. Mat., 30:1073-1100, 1966.

[17] Igor V. Dolgachev. Classical algebraic geometry. Cambridge University Press, Cambridge, 2012. A modern view.

[18] Shmuel Friedland and John Milnor. Dynamical properties of plane polynomial automorphisms. Ergodic Theory Dynam. Systems, 9(1):67-99, 1989.

[19] M. H. Gizatullin. Rational G-surfaces. Izv. Akad. Nauk SSSR Ser. Mat., 44(1):110-144, 239, 1980.

[20] M. H. Gizatullin. The decomposition, inertia and ramification groups in birational geometry. In Algebraic geometry and its applications (Yaroslavl', 1992), Aspects Math., E25, pages 39-45. Friedr. Vieweg, Braunschweig, 1994.

[21] Julien Grivaux. Parabolic automorphisms of projective surfaces (after M. H. Gizatullin). Mosc. Math. J., 16(2):275-298, 2016.

[22] Julien Grivaux. Infinitesimal deformations of rational surface automorphisms. Math. Z., 288(3-4):1195-1253, 2018.

[23] V. A. Iskovskikh and I. R. Shafarevich. Algebraic surfaces [ MR1060325 (91f:14029)]. In Algebraic geometry, II, volume 35 of Encyclopaedia Math. Sci., pages 127-262. Springer, Berlin, 1996.

[24] Stéphane Lamy and Susanna Zimmermann. Signature morphism for the Cremona group over a non-closed field. J. EMS, (to appear), 2019.

[25] William E. Lang. Configurations of singular fibres on rational elliptic surfaces in characteristic two. Comm. Algebra, 28(12):5813-5836, 2000. Special issue in honor of Robin Hartshorne.

[26] Yu. I. Manin. Cubic forms, volume 4 of North-Holland Mathematical Library. NorthHolland Publishing Co., Amsterdam, 1986.

[27] Jean-Pierre Serre. Arbres, amalgames, $\mathrm{SL}_{2}$. Société Mathématique de France, Paris, 1977. Avec un sommaire anglais, Rédigé avec la collaboration de Hyman Bass, Astérisque, No. 46.

[28] N.I. Shepherd-Barron. Some effectivity questions for plane Cremona transformations. arxiv:1311.6608v5, 2019.

[29] A.S. Smogorzhevsky. Lobachevskian geometry. Little Mathematics Library. Mir Publishers, Second edition, 1982.

[30] Susanna Zimmermann. The Abelianization of the real Cremona group. Duke Math. J., 167(2):211-267, 2018. 
Serge Cantat, Vincent Guirardel, Univ Rennes, CNRS, IRMAR - UMR 6625 , F-35000 RENNES, FRANCE

E-mail address: serge. cantat@univ-rennes $1 . \mathrm{fr}$

E-mail address: vincent.guirardelduniv-rennesl.fr

ANNE LONJOU, UNIVERSITY OF BASEL

E-mail address: anne.lonjou@unibas.ch 